\title{
Particulate Material Fabric Characterization by Rotational Haar Wavelet Transform
}

\author{
Junxing Zheng and Roman D. Hryciw
}

\begin{abstract}
A Rotational Haar Wavelet Transform (RHWT) method is developed to characterize the fabric of particulate assemblies from two-dimensional images. A Maximum Energy Ratio $\Psi$ reveals the fabric direction and its intensity. The method is implemented on 12 sand and 3 rice specimens of various shapes. It was shown that $\Psi$ may be expressed in terms of a material's aspect ratio and relative density. A material fabric classification system based on $\Psi$ is proposed. The parameter also defines the fabric tensor for cross anisotropic material. Scanning electron microscope images of kaolinite clay and several rock images are also analyzed.
\end{abstract}

\section{KEY WORDS}

Soil fabric, particle shape, image analysis, wavelet transform, sand 


\section{Introduction}

Because of depositional processes, fabric cross anisotropy (or transverse isotropy) is commonly encountered in earth materials [1-4]. Within the depositional plane, the soil particles' long axes point in random directions thus developing an isotropic fabric within that plane. This contrasts with the fabric established in the direction of deposition. The fabric anisotropy endows earth materials with macroscopic properties including strength, dilatancy, permeability and compressibility that vary with direction. This distinguishes soil from other civil engineering materials such as steel and concrete.

Laboratory tests for investigating soil behavior in different fabric directions were first performed on clays, perhaps because it is relatively easy to prepare anisotropic clay specimens by sampling intact blocks in different directions. The early works of Hansen and Gibson [5], Mitchell [6], Jacobsen [7], Lo [8], and Duncan and Seed [9] all showed that the shear strength of clays varies with the angle between the major principal stress and the deposition plane. Following these early studies, the influence of fabric anisotropy on strength, permeability, and compressibility of clay were systematically studied by Lo and Milligan [10], Saada [11], Lo and Morin [12], Saada and Ou [13], Prevost [14], Yong and Silvestri [15], Graham and Houlsby [16], Kirgard and Lade [2], Dewhurst et al. [17], Kurukulasuriya et al. [18], Nishimura et al. [19], Anantanasakul et al. [20] and many others.

Laboratory studies of the macro behavior of sands at different fabric directions began in the 1970s. It is somewhat difficult to prepare anisotropic sand specimens with varying fabric directions. One way is to freeze a wet specimen or impregnate an air-pluviated specimen with a resin to maintain its fabric and then trim the specimen in different orientations [21-22]. Another way is by using devices that incline a test vessel at various angles during air pluviation [23-25]. Following one of these specimen preparation procedures, various laboratory tests have been performed to study the influence of fabric anisotropy on macro behavior of sands. Studies have included plane strain compression tests by Oda et al. [22], Oda 
[26], and Tatsuoka et al. [23]; direct shear tests by Azami et al. [27], Guo [24], and Tong et al. [25]; triaxial tests by Oda [21], Arthur and Menzies [28], Arthur and Phillips [29], Wong and Arthur [30], Arthur et al. [31], Oda et al. [22], Lam and Tatsuoka [32], and Ochiai and Lade [33]; torsion shear tests by Rodriguez and Lade [34] and Yang et al. [35]. The influence of fabric anisotropy on bearing capacity and seismic response of foundations was studied by Meyerhof [36], and Oda and Koishikawa [37]. Besides physical laboratory tests, the effects of fabric anisotropy were also observed in Discrete Element Method (DEM) studies such as in the works of Hosseininia [38], Ng [39], Zhao and Guo [40], Fu and Dafalias [41]. Yimsiri and Soga [42]. Based on these laboratory and numerical observations, constitutive models that accounted for fabric anisotropy were developed by Pietruszczak and Mroz [43-44], Li and Dafalias [45], Dafalias et al. [46], Lade [47-48], Schweiger[49], Liu and Indraratna [50], Yao and Kong [51], Kong et al. [52], and Gao and Zhao [53].

Despite an abundance of evidence regarding the importance of fabric anisotropy on mechanical soil behavior, fabric anisotropy is rarely considered in routine geotechnical design and analysis. The primary reason is the lack of an easy, effective and accurate method to quantify it. The fabric anisotropy should be quantified from two aspects, the primary fabric direction and the degree of anisotropy. These two parameters can alternatively be computed based on the orientations of non-spherical particle long axes, from the orientations of interparticle contacts and, or the orientations of non-spherical voids (Oda and Nakayama [1]). The fabric computed from the orientations of non-spherical particle long axes is the focus of this study. This is the most frequently used fabric definition is the because it can be readily determined by visual observation. For example, Oda [21] impregnated resin into sand specimens then cut them into thin sections of thickness between 0.04 and $0.07 \mathrm{~mm}$. He manually measured the long axis orientations of 200 particles to determine fabric orientation. Oda's [21] method was later augmented by digital image processing techniques. Yang et al. [56] cut specimens at different locations (but not thin sections). They polished the surfaces before capturing images. Fonseca et al. [54, 55] captured the 
section images of specimens using micro-computed tomography. Instead of manually counting particle orientations, Yang et al. [56] and Fonseca et al. [54, 55] used image thresholding techniques to identify individual particles and compute the orientation distributions of particle long axes. Requisite to this method is that sand particles must have a different color from the surrounding resin. The greatest challenge is to computationally determine the boundaries of contacting particles so that their orientations could be assessed individually.

Existing fabric and anisotropy quantification methods are all based on statistical distributions of individual particles' long axes. Although it is well known that elongated particles will develop stronger fabric anisotropy than spherical particles, particle shape is not typically considered. Guo (2008) showed that direct shear strength systematically increases as particle elongation increases from perfectly round glass beads to sub-rounded Ottawa sand to angular crushed limestone. Similar observations were made by Oda [26], Lade[48], and Tong et al. [25]. Therefore, complete fabric characterization should logically include particle elongation.

To meet the challenge of simultaneously characterizing and quantifying the fabric and shape of particles from images, a new Rotational Haar Wavelet Transform (RHWT) technique is proposed in this paper. Instead of identifying individual particles, the RHWT analyzes grayscale distributions and their changes across an image. This allows the method to be utilized on images of contacting particle assemblies; although, it could just as well be used on individual particles. Images of clays and rock will also be analyzed to demonstrate the method's ability to characterize fabric in other earth materials. Finally, in recognition of the wide use of fabric tensors in soil mechanics and constitutive modeling, a method for determining the fabric tensor using the Rotational Haar Wavelet Transform will be presented. 


\section{Haar Wavelet Transform}

The Haar Wavelet Transform (HWT) has been used extensively for digital image compression, denoising, edge detection, feature extraction, texture analysis and image segmentation. The HWT is also not new in geotechnical engineering. It has been used to determine particle size distributions by Shin and Hryciw [57], Hryciw et al. [58] and Ohm and Hryciw [59], and for analysis of surface roughness by Chandan et al. [60]. The rigorous mathematical formulation of the HWT is detailed in many textbooks and will not be repeated here. However, the authors will illustrate the essence of the HWT through a simple example that will illustrate why the HWT is ideally suited for analysis of fabric anisotropy.

The example is the idealized $8 \times 8$ pixel grayscale image $A_{0}$ shown in Fig. 1 . The larger grayscale values shown correspond to brighter areas in the image. They change only in the horizontal direction while staying constant vertically. This reflects a strongly vertical fabric.

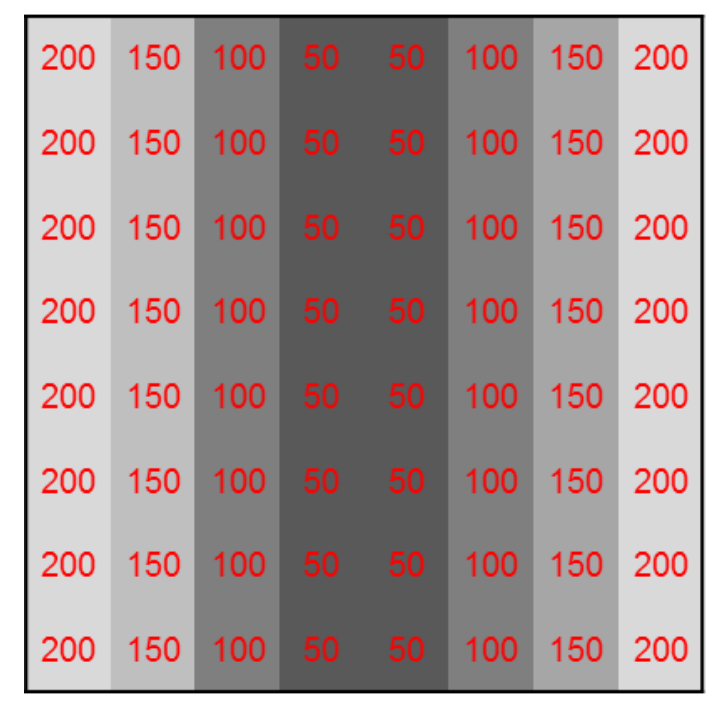

Fig. 1. An $8 \times 8$ intensity image with a strongly vertical fabric.

The HWT first divides the image into $2 \times 2$ subareas as shown in Fig. 2(a). The location of each $2 \times 2$ subarea can be represented by $A_{0}(i, j)$ where $i$ and $j$ range from 1 to 4 .

Assuming the four numbers in an $(i, j)$ region are: 


$$
A_{0}(i, j)=\left[\begin{array}{ll}
a & b \\
c & d
\end{array}\right]
$$

the HWT computes four values $A_{1}(i, j), H_{1}(i, j), V_{1}(i, j)$, and $D_{1}(i, j)$. The $A_{1}(1,1)$ is twice the average of the four numbers:

$$
A_{1}(i, j)=\frac{a+b+c+d}{2}
$$

The $H_{1}(i, j)$ is the average difference between two columns:

$$
H_{1}(i, j)=\frac{(a+c)-(b+d)}{2}
$$

The $V_{1}(i, j)$ is the average difference between two rows:

$$
V_{1}(i, j)=\frac{(a+b)-(c+d)}{2}
$$

The $D_{1}(i, j)$ is the average difference between two diagonals:

$$
D_{1}(i, j)=\frac{(a+d)-(b+c)}{2}
$$
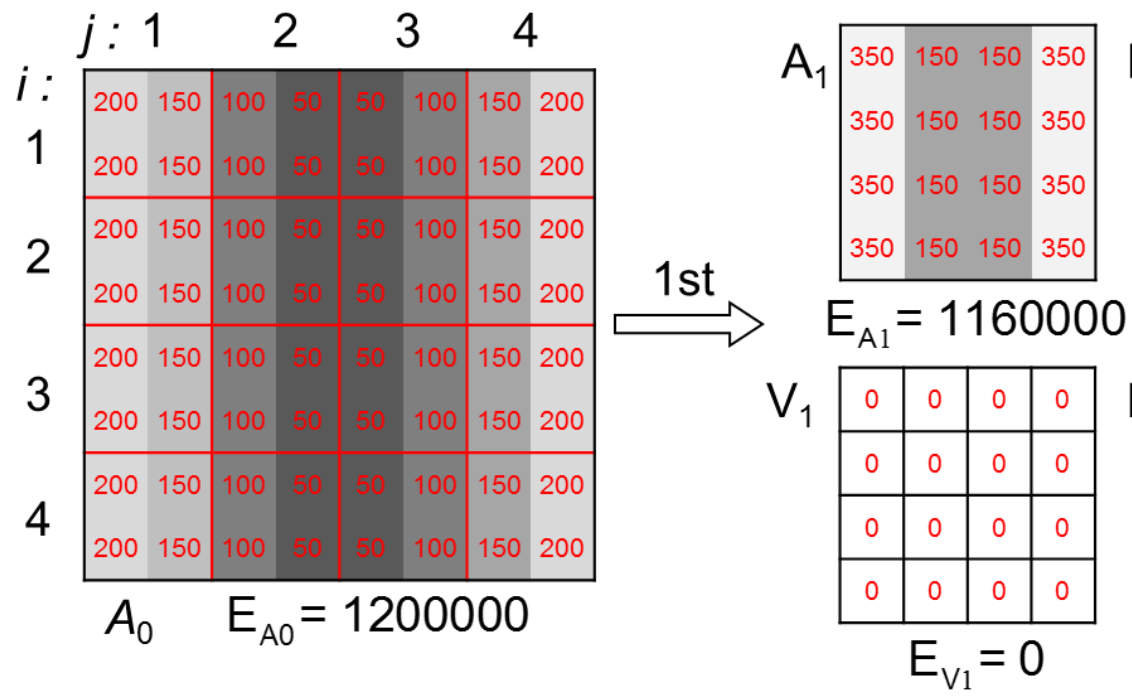

\begin{tabular}{c|c|c|c|c|}
$\mathrm{H}_{1}$ & 50 & 50 & -50 & -50 \\
\cline { 2 - 5 } & 50 & 50 & -50 & -50 \\
\hline 50 & 50 & -50 & -50 \\
\hline 50 & 50 & -50 & -50 \\
\hline
\end{tabular}

$E_{\mathrm{H} 1}=40000$

$\mathrm{D}_{1}$

\begin{tabular}{|l|l|l|l|}
\hline 0 & 0 & 0 & 0 \\
\hline 0 & 0 & 0 & 0 \\
\hline 0 & 0 & 0 & 0 \\
\hline 0 & 0 & 0 & 0 \\
\hline
\end{tabular}

$\mathrm{E}_{\mathrm{D} 1}=0$

Fig. 2. The $1^{\text {st }}$ Haar wavelet transform decomposition. 
For our example, the computed results for the first subarea $A_{0}(1,1)$ are: $A_{1}(1,1)=350, H_{1}(1,1)=50, V_{1}(1$, 1) $=0$, and $D_{1}(1,1)=0$. These computations are repeated for all 16 subareas which yield the $4 \times 4$ matrices $A_{1}, H_{1}, V_{1}$, and $D_{1}$ shown in Fig. 2. Only $A_{1}, H_{1}$, and $V_{1}$ are useful for fabric characterization. The matrix $A_{1}$ (which can be thought of as a $4 \times 4$ image) is essentially a downscaling of $A_{0}$ by a factor of 2 . The matrix $H_{1}$ quantifies the grayscale change in the horizontal direction while $V_{1}$ quantifies the grayscale change in the vertical direction. Since there is no grayscale change in the vertical direction $V_{1}$ is a zero matrix.

The energy $(E)$ is defined as the sum of the squares of values in a matrix and are shown beneath each matrix in Fig. 2. The energy of the original image is preserved in the four submatrices $A_{1}, H_{1}, V_{1}$, and $D_{1}$ in a HWT:

$$
E_{A 0}=E_{A 1}+E_{H 1}+E_{V 1}+E_{D 1}
$$

A relatively high $E_{\mathrm{H} 1}$ (or $E_{\mathrm{V} 1}$ ) would indicate the total differences in grayscale values in the horizontal (or vertical) direction is large in the original image $A_{0}$.

The HWT can now be applied to $A_{1}$ as shown in Fig. 3(a). This is the second decomposition of $A_{0}$. It yields another four submatrices $A_{2}, H_{2}, V_{2}$ and $D_{2}$. As before, $A_{2}$ is a downscaling of $A_{1}$ by a factor of 2 . The $H_{2}$ and $V_{2}$ quantify the grayscale changes in the horizontal and vertical directions in $A_{1}$ respectively. The $A_{2}$ can be further decomposed into $A_{3}, H_{3}, V_{3}$ and $D_{3}$ as shown in Fig. 3(b). Again, the energy is preserved at each decomposition. The first, second, and third decompositions are together called a three-level HWT. The maximum number of decomposition levels depends on the original image size. For a $2^{L} \times 2^{L}$ image, $L$ decompositions can be performed. At the i-th decomposition level, the computed energy $E_{\mathrm{Hi}}$ and $E_{\mathrm{Vi}}$ quantify the total differences in grayscale values in the horizontal and vertical directions in the $A_{\mathrm{i}-1}$ image. After all of the decompositions are performed, we define the maximum $E_{\mathrm{Hi}}$ and the maximum $E_{\mathrm{Vi}}$ as the horizontal and vertical energy $E_{\mathrm{H}}$ and $E_{\mathrm{V}}$ of the image: 


$$
\begin{gathered}
E_{H}=\max _{i=1 \ldots L}\left(E_{H i}\right) \\
E_{V}=\max _{i=1 \ldots L}\left(E_{V i}\right)
\end{gathered}
$$

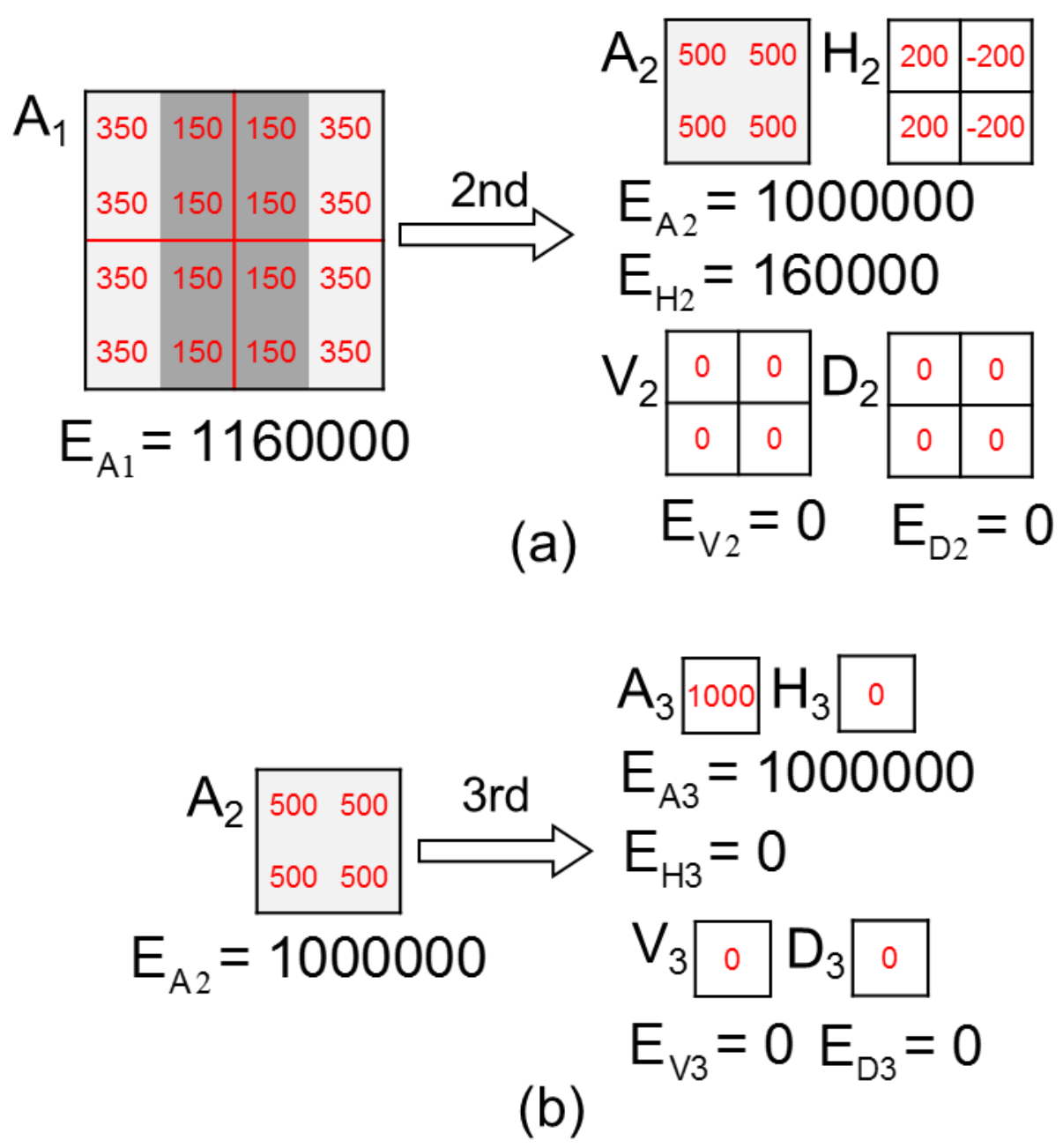

Fig. 3. The 2nd and 3rd Haar wavelet transform decompositions.

In the above example, $E_{\mathrm{H}}$ and $E_{\mathrm{V}}$ are 160,000 and 0 respectively. The physical meaning of Eqs. (7) and (8) will be discussed shortly.

The material fabric is associated with grayscale changes in an image. When there is very little grayscale change in a direction, the energy in this direction will be small. For example, the $E_{\mathrm{V}}$ of $A_{0}$ is zero in our 
example and this reveals and defines the fabric direction. Meanwhile, perpendicular to the fabric direction, the grayscale changes should be largest and thus produce the maximum energy. As such, to determine fabric direction, we search for the direction that exhibits the minimum energy and the maximum energy perpendicular to it. However, the HWT computes energy only in the horizontal and vertical directions in an image and therefore cannot identify fabric in other directions. Therefore, a Rotational Haar Wavelet Transform (RHWT) is proposed to determine energy values in all directions.

\section{Rotational Haar Wavelet Transform}

A synthetic image $M$ was generated having a fabric direction at $60^{\circ}$ from the horizontal as shown in Fig. 4(a). Two energy terms $E(\theta)$ and $E\left(\theta+90^{\circ}\right)$ are now defined: $E(\theta)$ is the energy in the direction at an angle of $\theta$ counterclockwise from the horizontal; it quantifies the grayscale change in the $\theta$ direction while $E\left(\theta+90^{\circ}\right)$ quantifies the grayscale change perpendicular to the $\theta$ direction. To identify the fabric orientation, we seek the $\theta$ value that simultaneously yields the minimum $E(\theta)$ and the maximum $E\left(\theta+90^{\circ}\right)$. We now also define an energy ratio $E R$ as:

$$
E R(\theta)=\frac{2}{\pi} \arctan \left(\frac{E\left(\theta+90^{\circ}\right)}{E(\theta)}\right)
$$

The value of $E R$ will range from 0 to 1 . The $\theta$ corresponding to the maximum $E R$ is the fabric orientation. It is noted that this maximum $E R$ will be in the direction of minimum $E$. The need for creating this seeming contradiction in terminology will become apparent later.

To compute $E(\theta)$ and $E\left(\theta+90^{\circ}\right)$ and thus $E R(\theta)$, the original image $M$ may be thought of as being rotated clockwise by an angle $\theta$ as shown in Fig. 4(b). Then, a nine-level HWT is performed on a central $512 \times$ 512 pixel area of the image. The $E_{\mathrm{H}}$ and $E_{\mathrm{V}}$ are the maximum $E_{\mathrm{Hi}}$ and $E_{\mathrm{Vi}}$ following Eqs. (7) and (8). If we 
rotate $M$ back to its original position in Fig. $4(\mathrm{c})$, the $E(\theta)$ and $E\left(\theta+90^{\circ}\right)$ will be equal to $E_{\mathrm{H}}$ and $E_{\mathrm{V}}$ from the previously rotated view in Fig. $4(\mathrm{~b})$. Therefore, the $E R$ in the $\theta$ and $\theta+90^{\circ}$ directions can be computed by:

$$
E R(\theta)=\frac{2}{\pi} \arctan \left(\frac{E\left(\theta+90^{\circ}\right)}{E(\theta)}\right)=\frac{2}{\pi} \arctan \left(\frac{E_{V}}{E_{H}}\right)
$$

and

$$
E R\left(\theta+90^{\circ}\right)=\frac{2}{\pi} \arctan \left(\frac{E(\theta)}{E\left(\theta+90^{\circ}\right)}\right)=\frac{2}{\pi} \arctan \left(\frac{E_{H}}{E_{V}}\right)
$$

where $E_{\mathrm{H}}$ and $E_{\mathrm{V}}$ are obtained in the rotated image view in Fig. 4(b).

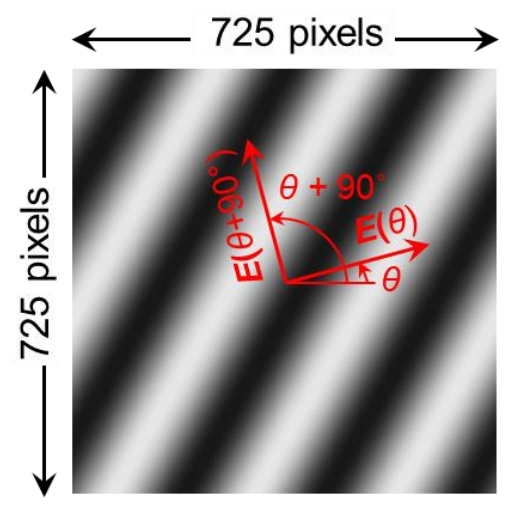

(a)

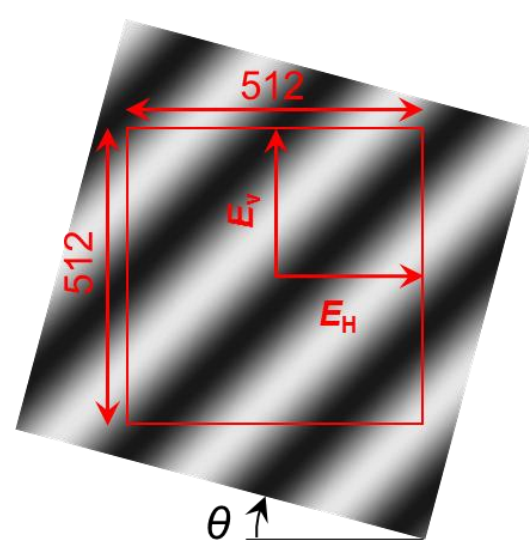

(b)

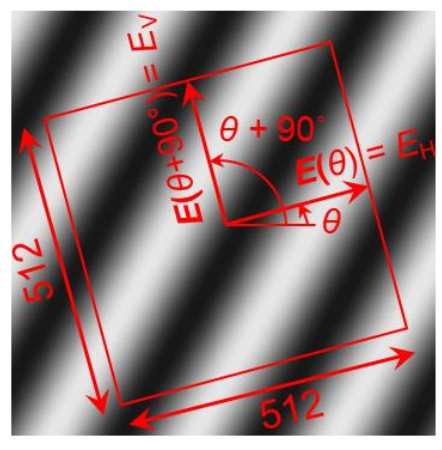

(c)

Fig. 4. The rotational Haar wavelet transform on an Image $M$

If we rotate the image $M$ from $0^{\circ}$ to $89^{\circ}$ by increments of $1^{\circ}$, a total of 90 images will be generated. For each rotation, a nine-level HWT is performed in a central $512 \times 512$ area to compute $E_{\mathrm{H}}$ and $E_{\mathrm{V}}$. Taking the computed $E_{\mathrm{H}}$ and $E_{\mathrm{V}}$ into Eq. (10), the $E R$ for $0^{\circ} \leq \theta \leq 89^{\circ}$ can be computed. Taking the computed $E_{\mathrm{H}}$ and $E_{\mathrm{V}}$ into Eqs. (11), the $E R$ for $90^{\circ} \leq \theta \leq 179^{\circ}$ can be computed. The computed $E R$ versus $\theta$ is plotted 
in Fig. 5(a). Due to rotational symmetry, $E R\left(\theta+180^{\circ}\right)=E R(\theta)$. Therefore, we can plot $E R$ in polar coordinates over the full range $0^{\circ} \leq \theta \leq 360^{\circ}$ in Fig. $5(\mathrm{~b})$.
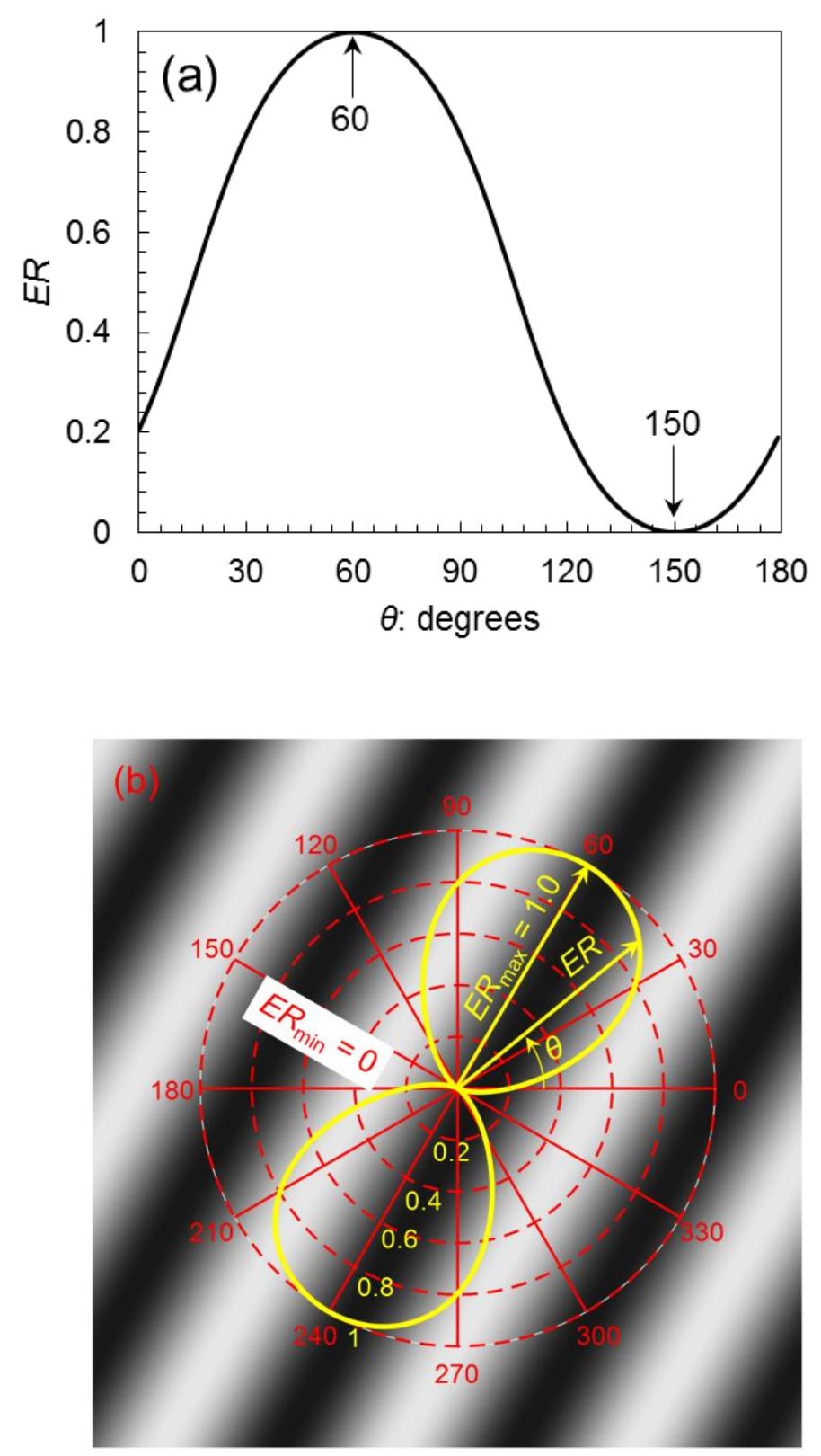

Fig. 5. The energy ratio of $M$ in different directions.

Fig. 5(b) explains why $E R$ was defined so that $E R_{\max }$ would be in the $E_{\min }$ direction. The $E R$ curve in Fig. $5(b)$ is equivalent to a rose diagram traditionally developed after statistically counting the number of 
particles oriented in each direction. The $E R$ values increase as $\theta$ approaches the fabric direction and the peak $E R\left(E R_{\max }\right)$ identifies the fabric orientation. Meanwhile, orthogonal to the fabric direction, $E R$ will be at a minimum $\left(E R_{\min }\right)$.

An important property of Eqs. (10) and (11) is that the sum of the computed $E R$ values in any two orthogonal directions equals 1 :

$$
E R(\theta)+E R\left(\theta+90^{\circ}\right)=1
$$

Therefore, the $E R_{\max }+E R_{\min }=1$ will also always be true.

We can anticipate that for a soil displaying isotropic fabric, the $E R$ plot will be a circle. This is verified by the synthetic image $S$ shown in Fig. $6($ a) which has the same grayscale changes in all direction. The image has a size of $724 \times 724$ pixels and a nine-level RHWT was performed on a central $512 \times 512$ pixel area. The computed $E R$ versus $\theta$ is plotted in Fig. $6(\mathrm{~b})$. As expected, the plot is a circle of radius $=0.5$ because $E_{\mathrm{H}}$ must be equal to $E_{\mathrm{V}}$ for all $\theta$.

(a)

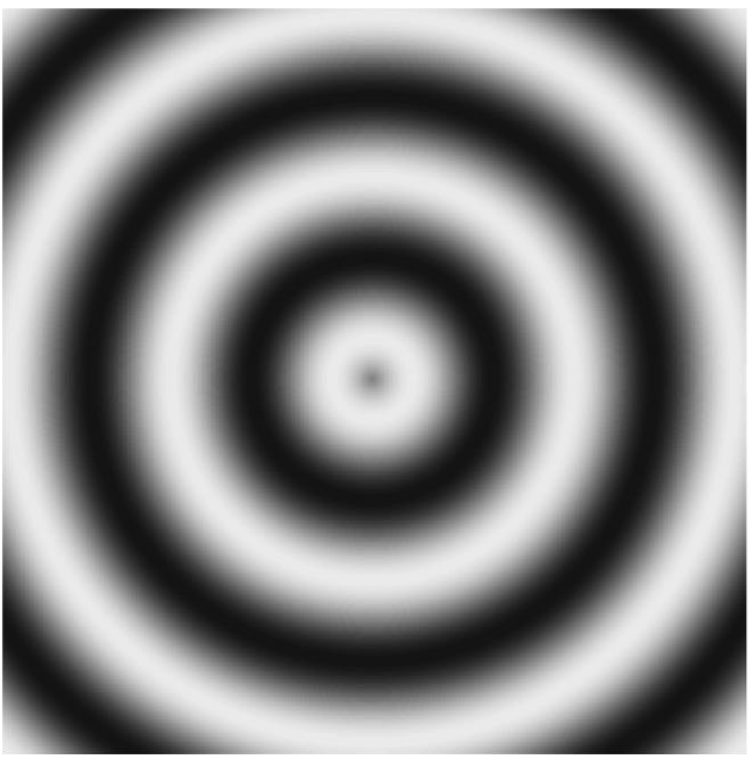

(b)

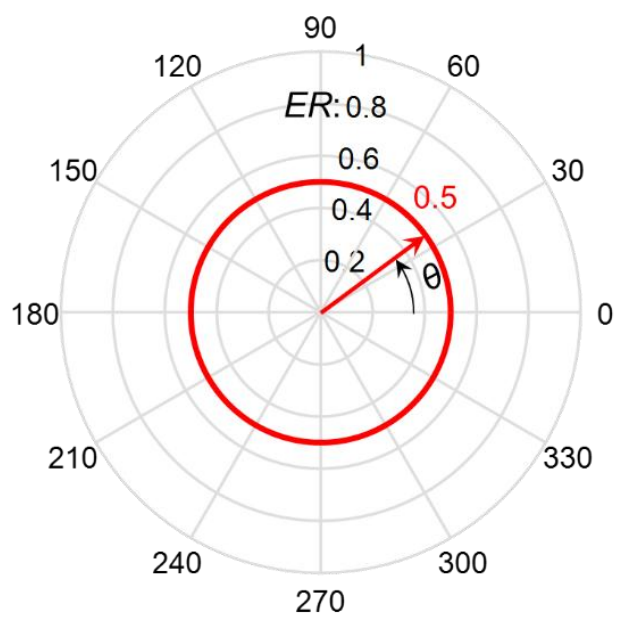

Fig. 6. The energy ratio for an image $S$ having an isotropic fabric. 
The two idealized images $M$ and $S$ represent two extreme fabric scenarios. Image $M$ has a completely anisotropic fabric and Image $S$ has a completely isotropic fabric. The RHWT clearly identifies fabric structure for such idealized images. The following sections will explore RHWT results for images of natural particulate materials.

\section{Fabric quantification of natural particulate material images by RHWT}

Before studying real soil, long-grain rice was used to demonstrate the RHWT. The long-grain rice is used because it is very elongated and therefore the RHWT results will be very distinct. A typical long-grain rice image is shown in Fig. 7 . The image size is $1450 \times 1450$ pixels. The image was rotated from $0^{\circ}$ to $89^{\circ}$ in $1^{\circ}$ increments. At each rotation step, a ten-level HWT was performed on a central $1024 \times 1024$ pixel region. The analyzed regions at rotations $\theta=0^{\circ}, 22^{\circ}, 45^{\circ}$ and $67^{\circ}$ are shown in Fig. 7 .

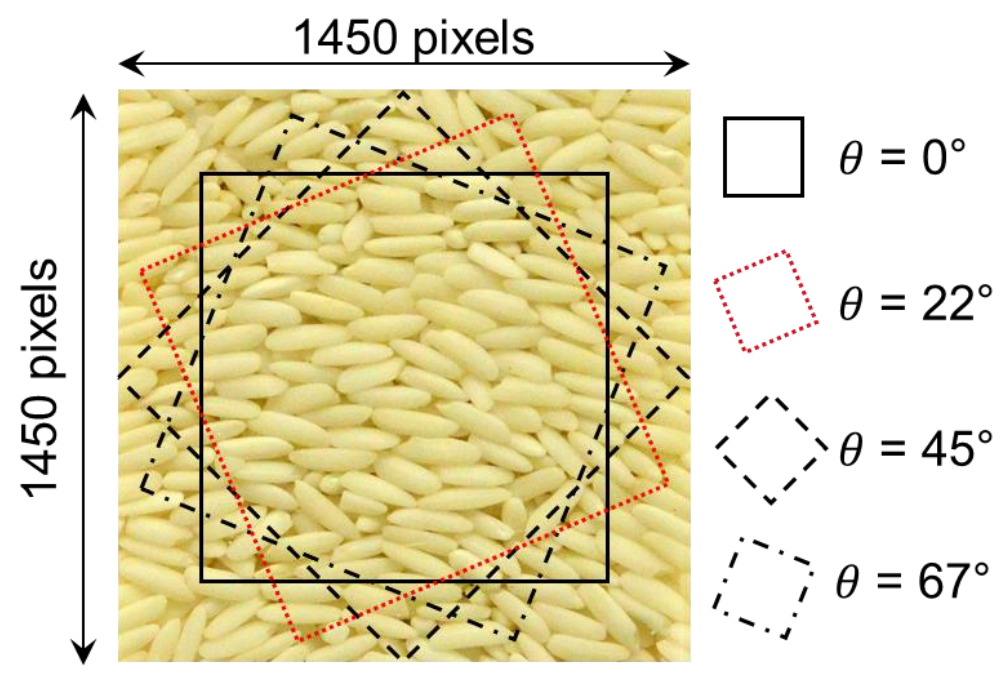

Fig. 7. A long-grain rice image and the analyzed regions. 
The HWT decompositions for $\theta=0^{\circ}$ are shown in Fig. 8. The $A_{0}$ is the original image while $A_{1}$ to $A_{7}$ are the first through seventh decompositions. The computed $E_{\mathrm{Hi}}$ and $E_{\mathrm{Vi}}$ values are plotted in Fig. 9. It should be noted that $E_{\mathrm{Hi}}$ and $E_{\mathrm{Vi}}$ quantify the horizontal and vertical grayscale changes in $A_{\mathrm{i}-1}$.



Fig. 8. The first seven HWT decompositions at $\theta=0^{\circ}$.

In Fig. 9, both $E_{\mathrm{Hi}}$ and $E_{\mathrm{Vi}}$ values of the long-grain rice display bell-shaped curves and have maximum values at $i=5$. The same trends have generally been observed for other natural particulate materials, although the maximum values may not be at $i=5$. Using the long-grain rice example, we will explain why both $E_{\mathrm{Hi}}$ and $E_{\mathrm{Vi}}$ follow bell-shaped curves and why in this example the maximum values are at $i=5$.

Image grayscale changes occur not only at the particle edges but also within particles interiors due to subtle material textures. However, these internal grayscale texture changes are much weaker than the grayscale changes at particle edges. As the original image is consecutively downscaled to smaller resolutions, the internal grayscale changes are gradually filtered out while the grayscale changes at edges become dominant as shown from $A_{0}$ to $A_{4}$ in Fig. 8. Correspondingly, both $E_{\mathrm{Hi}}$ and $E_{\mathrm{Vi}}$ increase and reach their peaks at $A_{4}$ meaning the majority of the internal texture is eliminated and the grayscale changes only occur at edges. At higher post-peak decomposition levels the grayscale changes at edges 
will also be filtered out. Thus, the entire image becomes progressively blurred as shown in $A_{5}$ to $A_{7}$. This reduces both $E_{\mathrm{Hi}}$ and $E_{\mathrm{Vi}}$. The maximum $E_{\mathrm{Hi}}$ and $E_{\mathrm{vi}}$ values essentially quantify the grayscale change in the horizontal and vertical directions only due to the occurrences of edges. Obviously, only the edges should define the material fabric. This is why Eqs. (7) and (8) use only the maximum $E_{\mathrm{Hi}}$ and $E_{\mathrm{vi}}$ values to define the horizontal and vertical energies $E_{\mathrm{H}}$ and $E_{\mathrm{V}}$.

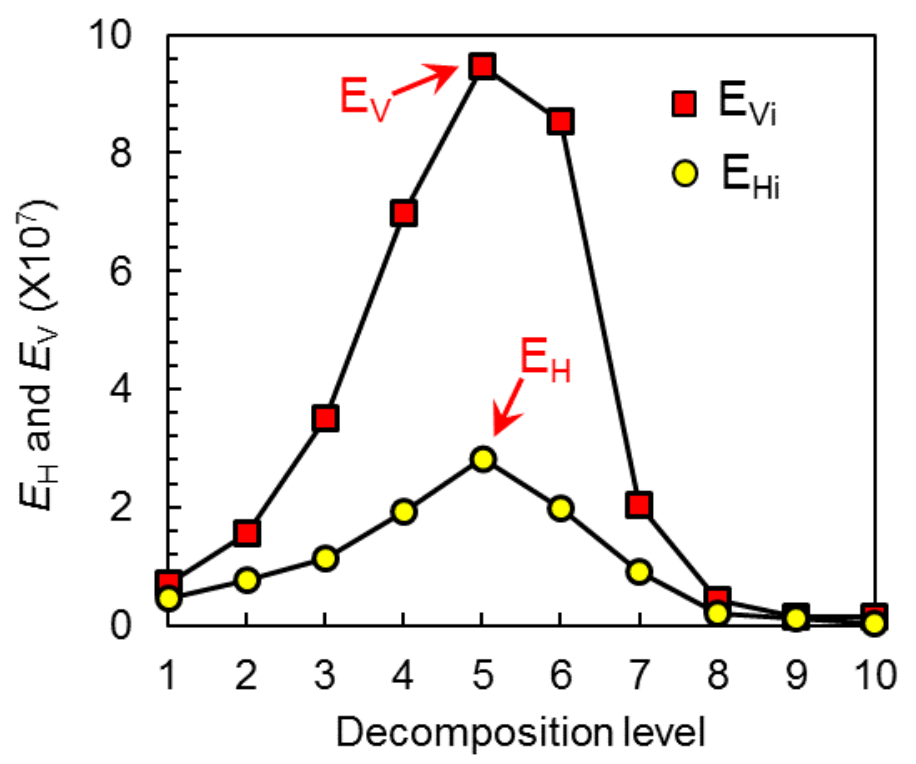

Fig. 9. The computed horizontal and vertical energies at $\theta=0^{\circ}$

In the synthetic images in Figs. 1 and 4, the grayscale did not change along the fabric direction, therefore the energy was zero in this direction. However, in images of natural materials such as Fig. 8, the grayscale will also change in the fabric direction due to the occurrence of short edge segments normal to the fabric direction. Nevertheless, the occurrence of long edges in the direction parallel to the fabric direction will be more frequent than the occurrence of short edges normal to the fabric direction.

For the long-grain rice example, the HWT was performed at each rotation angle and the resulting ER plot is shown in Fig. 10(a). It confirms a horizontal fabric orientation in agreement with a visual observation 
of Fig. 7. However, unlike for the previous perfect synthetic images $M$ and $S$, the $E R$ plot is zig-zagged because of the non-perfectly aligned particle orientations and somewhat due to image noise. To smooth out this "zig-zaggedness", a second order Fourier series is used to fit the ER curve:

$$
E R(\theta)=a_{0}+a_{1} \cos (\theta)+b_{1} \sin (\theta)+a_{2} \cos (2 \theta)+b_{2} \sin (2 \theta)
$$

Since $E R\left(\theta+180^{\circ}\right)=E R(\theta), a_{1}$ and $b_{1}$ must be zero. Equation (13) must also satisfy Eq. (12). Therefore, $a_{0}$ must be 0.5 . With these two observations, Eq. (13) simplifies to:

$$
E R(\theta)=0.50+a_{2} \cos (2 \theta)+b_{2} \sin (2 \theta)
$$

The coefficients $a_{2}$ and $b_{2}$ can be determined by fitting Eq. (14) to the original $E R$ plot as shown in Fig. 10(b). For the long-grain rice image the parameters were found to be: $a_{2}=0.350$, and $b_{2}=-0.077$. The $E R_{\max }$ and $E R_{\min }$ are computed as:

$$
\left[\begin{array}{l}
E R_{\max } \\
E R_{\min }
\end{array}\right]=0.50 \pm \sqrt{a_{2}^{2}+b_{2}^{2}}
$$

For the plot in Fig. 10 (b) $E R_{\max }=0.86$ and $E R_{\min }=0.14$. The fabric direction (direction of $E R_{\max }$ ) is $174^{\circ}$.

A rotational increment of $1^{\circ}$ was used in the above computation in above computation but other increments could also be used. Figs. 10 (c) and (d) show the results of using a rotational increment of $0.1^{\circ}$ while Figs. 10 (e) and (f) show the results for an increment of $10^{\circ}$. As the rotational increment increases, the original the original $E R$ plot becomes smoother. However, the computed Fourier plots are very close in all cases. The Fourier plots depend only on $a_{2}$ and $b_{2}$ as shown in Eq. (14). Therefore, despite having fewer $E R$ values computed using larger rotational increments, the $a_{2}$ and $b_{2}$ from regression are very close. This study found that once the rotational increment is smaller than $1^{\circ}$, the computed Fourier $E R$ plots are unaffected by the increment. Therefore, a rotational increment of $1^{\circ}$ is suggested. 


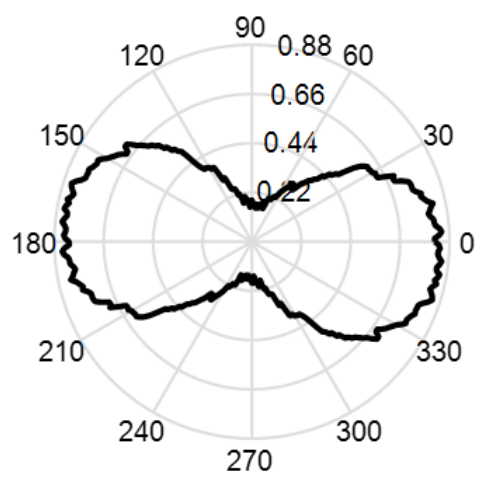

(a)

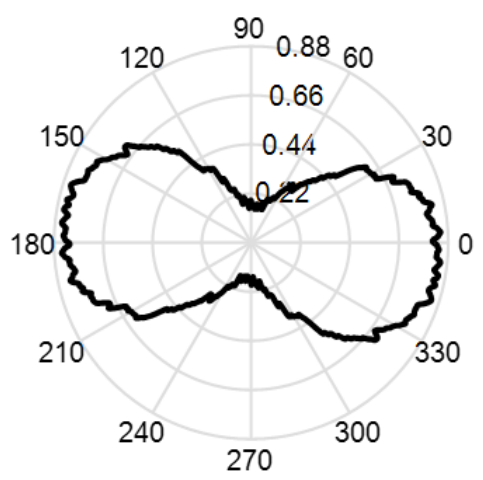

(c)

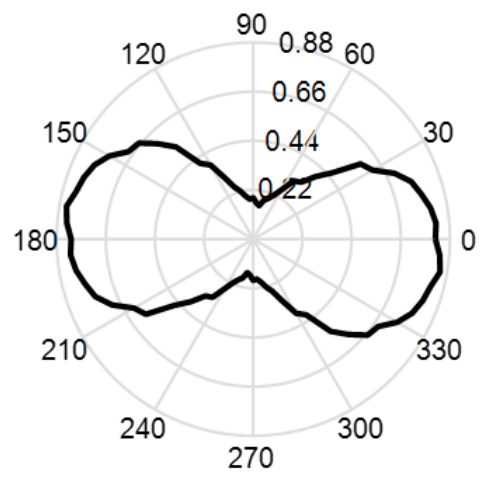

(e)

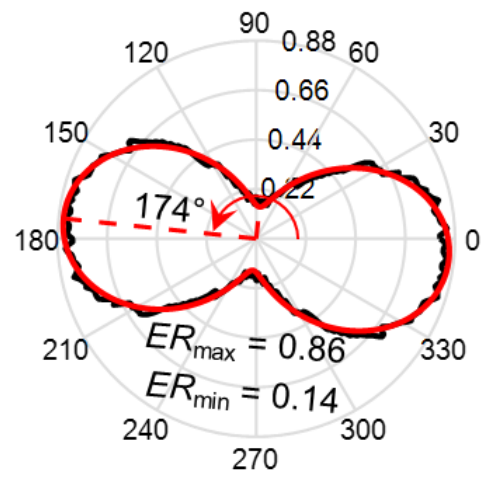

(b)

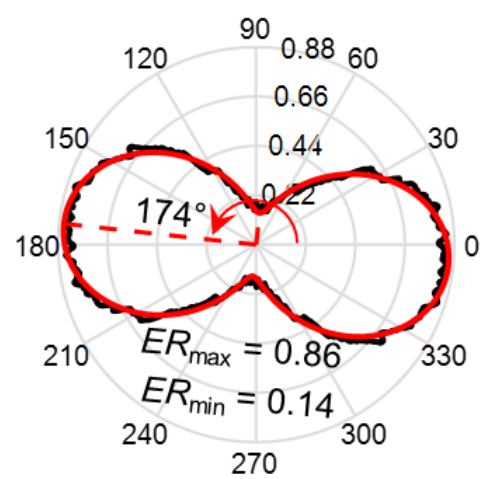

(d)

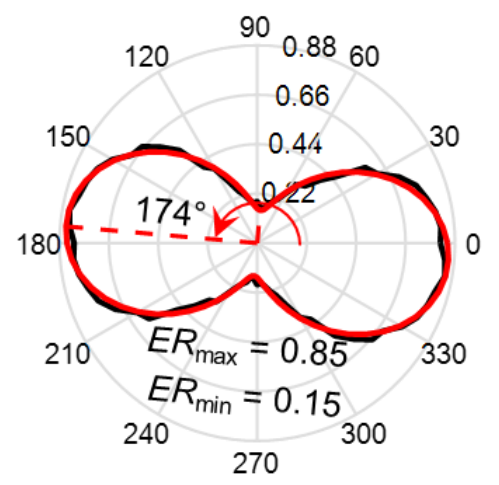

(f)

Original ER plot Fourier ER plot

Fig. 10. The original $E R$ data and the smoothed Fourier $E R$ plot for the long-grain rice in Fig. 7. 


\subsection{Effects of PPS on RHWT results}

In the previous example, the image size was $1450 \times 1450$ of which its central $1024 \times 1024$ area was analyzed. Generally, if the size of the analyzed central region is $2^{L} \times 2^{L}$ on which an L-level HWT is performed, the corresponding image size must be at least $2^{L+0.5} \times 2^{L+0.5}$. For example, if the analyzed central area is to be $128 \times 128$, the original image size must be at least $181 \times 181$. This raises an important question: what is the appropriate image magnification to ensure that accurate $E R$ values are obtained. The number of pixels corresponding to the shorter particle dimension is the appropriate criteria to study this problem. We refer to this value as the "pixels per smaller dimension" (PPS). For example, The PPS of the long-grain rice in Fig. 7 is about 55 pixels. For a fixed particle size, we can anticipate that too small a PPS will suppress the grayscale change at the particle edges (such as $A_{4}$ to $A_{7}$ in Fig. 8) and therefore the $E_{\mathrm{H}}$ and $E_{\mathrm{V}}$ will approach each other as shown in Fig. 9. Consequently, the degree of fabric anisotropy will be underestimated.

The long-grain rice can develop a strong fabric, Fig. 7 is used to investigate the effects of PPS. If we could determine the minimum PPS that would correctly characterize such a strong fabric anisotropy we would be able to use this minimum PPS as the criterion for other natural materials, such as sands, whose particles are less elongated. The long-grain rice image was upscaled and downscaled to generate a series of images having different PPS values. The images were $46 \times 46,91 \times 91,181 \times 181,362 \times 362,724 \times$ 724 , and $2897 \times 2897$ pixels. The analyzed central areas were correspondingly $2^{5} \times 2^{5}, 2^{6} \times 2^{6}, 2^{7} \times 2^{7}, 2^{8}$ $\times 2^{8}, 2^{9} \times 2^{9}$, and $2^{11} \times 2^{11}$ pixels. The corresponding PPS values are $2,4,7,14,28$, and 110 pixels.

The computed original and Fourier ER plots for different PPSs are shown in Fig. 11. The results are stable and consistent once PPS exceeds 7 pixels. Therefore, the required minimum PPS to achieve reliable results is 7 pixels. In some circumstances, large PPS images may be available using very high resolution 
devices. For such images, users can either use more powerful computers to implement the RHWT or simply downscale the image to an appropriate PPS value (must be larger than 7 pixels). Such downscaling does not change the computed fabric structure as shown in Fig. 11.

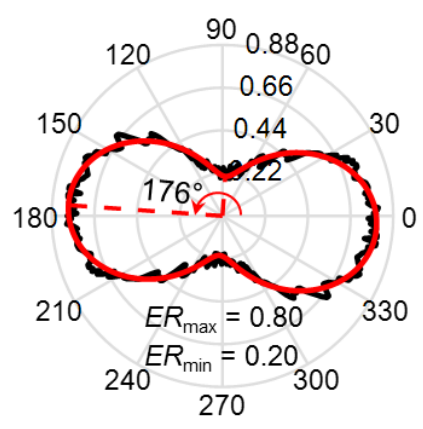

(a) $P P S=2$ pixels



(d) $P P S=14$ pixels

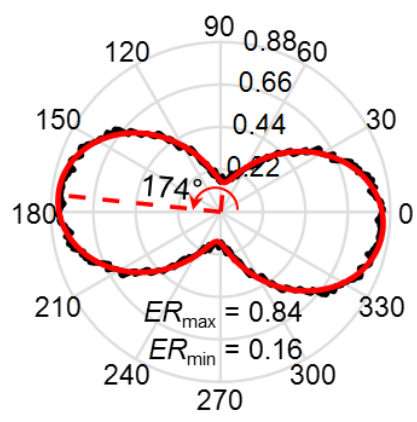

(b) $P P S=4$ pixels

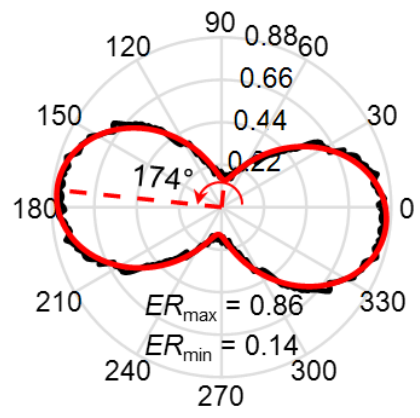

(e) $P P S=28$ pixels

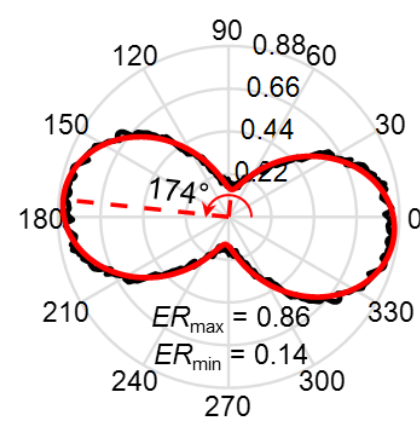

(c) $P P S=7$ pixels

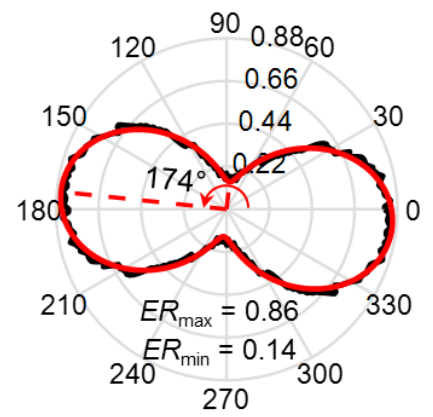

(f) $P P S=110$ pixels

Original ER plot Fourier ER plot

Fig. 11. Effect of image magnification on the computed $E R$ plot

For images of natural materials, the computed $E R$ plots must be somewhere between the two extreme cases shown in Figs. 5(b) and 6(b). The computed $E R_{\max }$ will be in the range between 0.5 and 1.0 while the computed $E R_{\min }$ will be between 0.0 and 0.5 . The degree of fabric anisotropy can be quantified by the shape of the $E R$ plot. Therefore, the $E R_{\max }$ can serve as a quantifiable metric of the degree of fabric anisotropy. For brevity, the $E R_{\max }$ is hereafter symbolized by $\Psi$. 


\section{Particulate material fabric by RHWT}

The degree of fabric anisotropy developed in particulate materials depends on both the orientation distribution of the particles' long axes and the particle Aspect Ratio $(A R)$. The $A R$ is defined as the ratio of particle length to particle width, which can be readily determined by computational geometry techniques proposed by Zheng and Hryciw [61] or by caliper measurements. The $A R$ is an intrinsic property that brackets the range of fabric anisotropies which a material can develop while the orientation distribution of particle long axes is a state property. Those two properties will be investigated in this section.

\subsection{Orientation distributions of particle long axes}

A series of long-grain rice specimens displaying different degrees of fabric anisotropy were prepared as shown in Figs. $12(\mathrm{a})$ to $(\mathrm{k})$. The image sizes were $1450 \times 1450$ pixels in which their central $1024 \times 1024$ pixel areas were analyzed by RHWT. The computed original and Fourier ER plots are shown in Figs. 12(b) to (I) in which the $\Psi$ values and fabric orientations are also provided. For reference, the dashed circles at $E R=0.5$ shown in each figure represent the isotropic fabric state. The computed results agree well with visual observations.

The particles in Fig. 12(a) display completely random orientations. Therefore, the computed ER plot overlaps the isotropic circle. From Fig. $12(b)$ to $(k)$, the long axes of the particles gradually manifest stronger preferred orientations. Therefore, the $E R$ plots sequentially depart from the isotropic circle and approach a strongly anisotropic fabric plot. The range of degrees of fabric anisotropy that were 
observed for the long-grain rice was from near perfect isotropy ( $\Psi=0.51$ ) in Fig. 12(a) to extreme anisotropy $(\Psi=0.86)$ in Fig. 7.
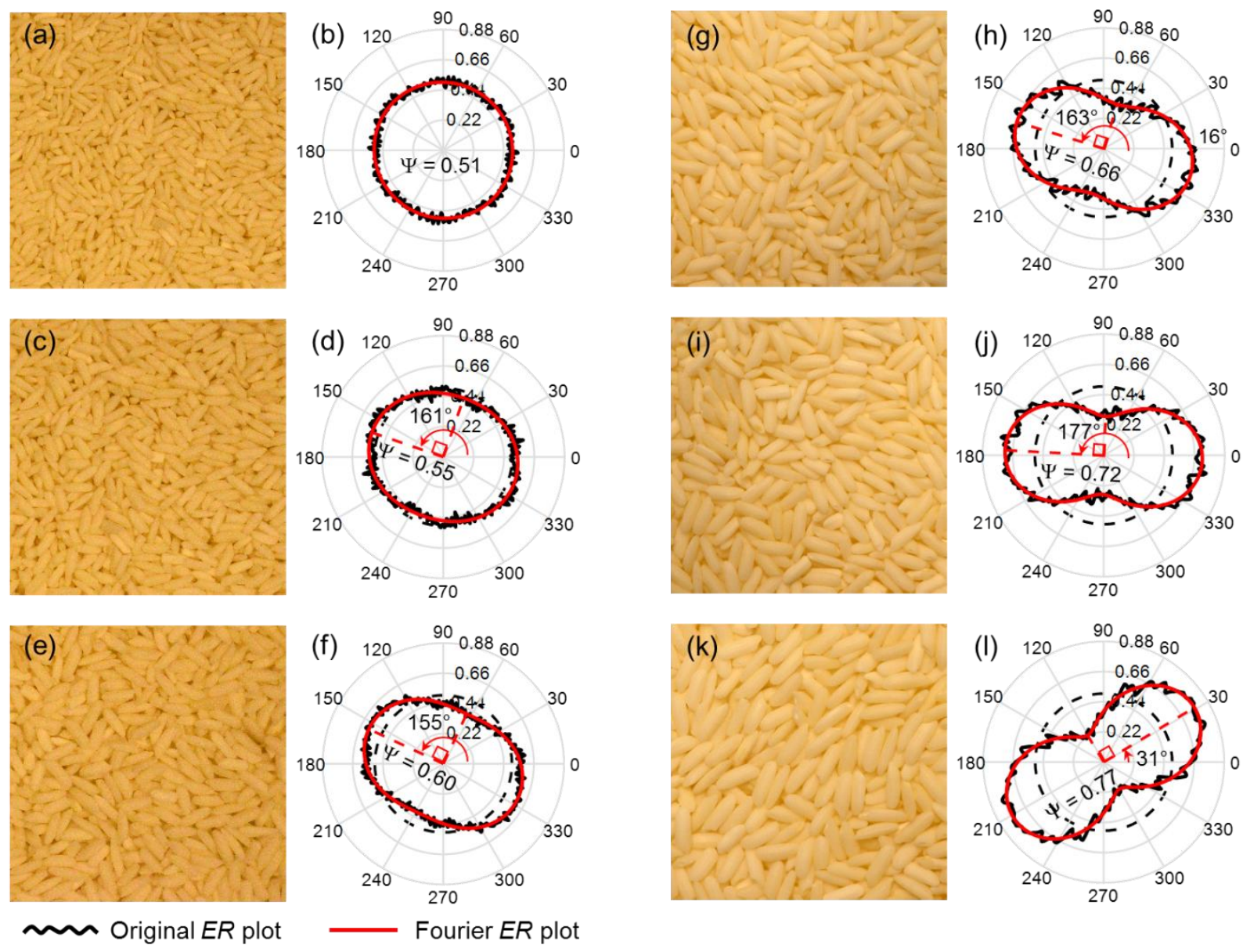

Fig. 12. A series of rice images displaying different degrees of fabric anisotropy.

\subsection{Particle aspect ratio}

The range of fabric anisotropy that can develop in a sand depends on the particle Aspect Ratio (AR). Particles having larger ARs tend to develop stronger fabric anisotropy and thus display a wider fabric range. Different sands with a range of $A R$ values will be tested to explore the relationship between $A R$ and degrees of fabric anisotropy. 
A total of 15 sands representing a range of particle sizes, gradations, roundnesses, aspect ratios and particle colors as listed in Table 1 were investigated. The particle size distributions were determined using sieving tests while the index void ratios were determined following ASTM D4253-14 [62] and ASTM D4254-14 [63]. For each soil, the average $A R$ value was computed after analyzing 200 particles using Zheng and Hryciw [61]'s computational geometry techniques. As shown in Table 1, the average $A R$ values of natural soils are in a relative narrow range of 1.22 (Ottawa Sand) to 1.78 (Crushed Gabbro). Zheng and Hryciw [64] collected the ARs of 167 sands (including natural sands and crushed rocks). They found that the average $A R$ s of most sands range between 1.2 and 1.8. More elongated soil particles are rare in nature because they are vulnerable to breakage. While occasional individual particles could have larger $A R$ values, it is rare for natural sands to possess average $A R$ values larger than 1.8 . As such, shortgrain rice, medium-grain rice, and long-grain rice were added to expand the studied $A R$ range.

Air pluviation was used to generate the cross-anisotropic fabric structure. The laboratory equipment included a $5 \mathrm{~cm} \times 5 \mathrm{~cm}$ square glass tube of $30 \mathrm{~cm}$ height, a funnel, a $2.5 \mathrm{~kg}$ weight for surcharge, a shaking table and a Nikon D800 camera with a magnification lens. The funnel and glass tube were used to prepare the loose specimen to replicate a previously determined $e_{\max }$. Images of $2900 \times 5800$ pixel size were captured through the side of the square glass tube. The captured images of five typical sands including Ottawa, Indiana Beach, Crushed Gabbro, short-grain rice and medium-grain rice are shown in Figs. $13(a)$ to $17(a)$. The glass tube was mounted on a shaking table and a surcharge weight was placed on top of the material. The specimens were vibrated until they achieved their respective independently established ASTM values of $e_{\min }$. Images were again captured after densification to $e_{\min }$. They are shown in Figs. 13(b) to 17(b).

To generate isotropic fabrics, the particles from each specimen were spread on a flat surface to ensure that the long axes of particles were randomly oriented. The images were captured from above the specimens. They are shown in Figs. 13(c) to 17 (c). The image sizes were also $2900 \times 5800$ pixels. 
The captured images were divided into 8 sub-images of $1450 \times 1450$ pixel size. For each sub-image, a ten decomposition level RHWT was performed on its central $1024 \times 1024$ pixel region. The horizontal and vertical energies of the original images at the $i$-th decomposition levels were averaged from all eight sub-squares:

$$
E_{H i}(\theta)=\frac{1}{m} \sum_{j=1}^{m} E_{H i, j}(\theta)
$$

and

$$
E_{V i}(\theta)=\frac{1}{m} \sum_{j=1}^{m} E_{V i, j}(\theta)
$$

where $m$ is the total number of sub-squares; $E_{\mathrm{Hi}, j}(\theta)$ and $E_{\mathrm{Vi}, j}(\theta)$ are the horizontal and vertical energy of the $j$-th sub-square at rotation $\theta$. Combining Eqs. (16), (17), (7), (8), (10) and (11), the $E R(\theta)$ of the original image was computed and fitted with a Fourier ER plot. Only the Fourier $E R$ plots are shown in Figs. $13(d)$ to 17 (d) in which the isotropic circle is also shown for reference. The original $E R$ plots are close to their fitted Fourier plots and therefore are not shown. The computed degrees of fabric in loose condition, dense condition and, flat surface tests are represented by $\Psi_{L}, \Psi_{\mathrm{D}}$, and $\Psi_{\mathrm{F}}$ respectively. The fabric directions of the loose and dense conditions are also shown.

As expected, the $E R$ plots of the flat surface tests were very close to the reference isotropic circles. The $E R$ plots for the dense condition display stronger anisotropy than the plots for the loose condition. The fabric directions for both the dense and loose conditions are very close to the horizontal direction. The $\Psi_{L}, \Psi_{D}$, and $\Psi_{F}$ values of all the soils are summarized in Table 1 . 


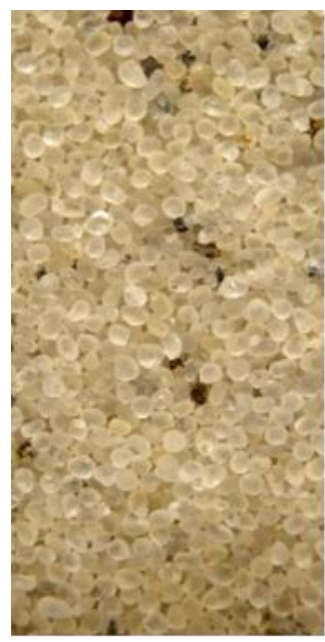

(a)

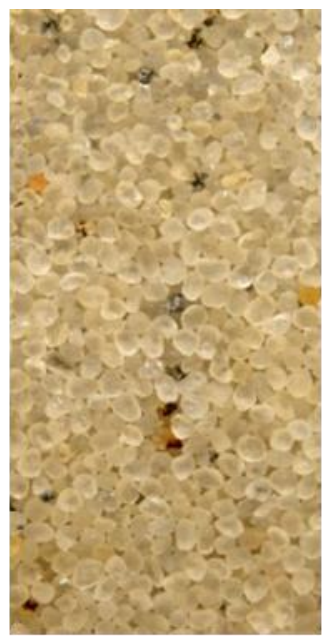

(b)



(c)

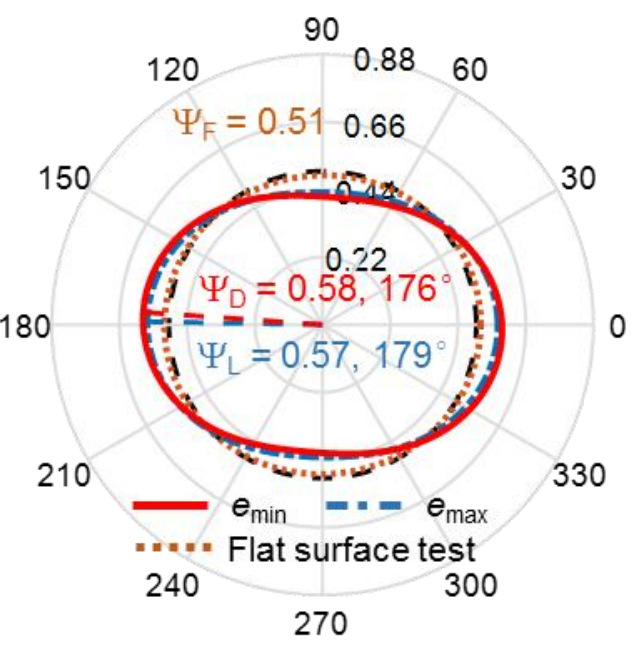

(d)

Fig. 13. Fabric anisotropy of Ottawa sand: (a) loose condition, (b) dense condition, (c) flat surface test, (d) computed ER plots.

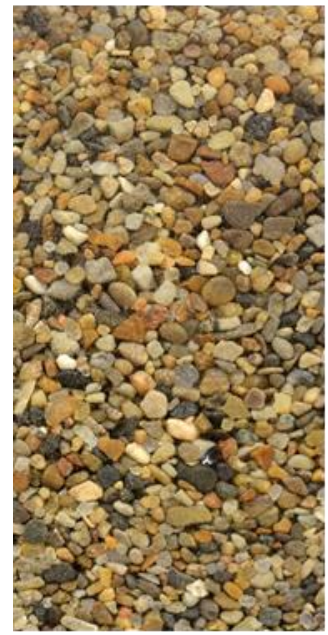

(a)

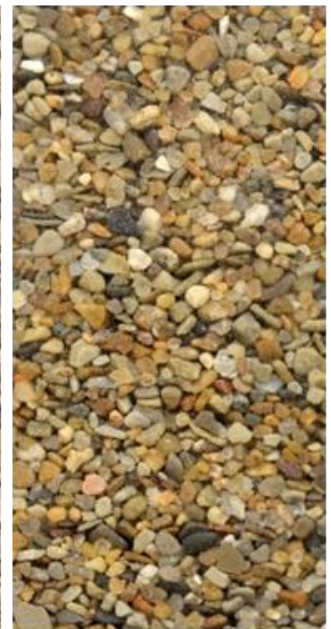

(b)

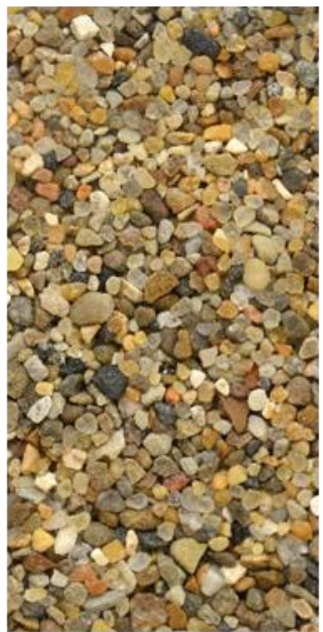

(c)



(d)

Fig. 14. Fabric anisotropy of Indiana Beach sand: (a) loose condition, (b) dense condition, (c) flat surface test, (d) computed ER plots. 


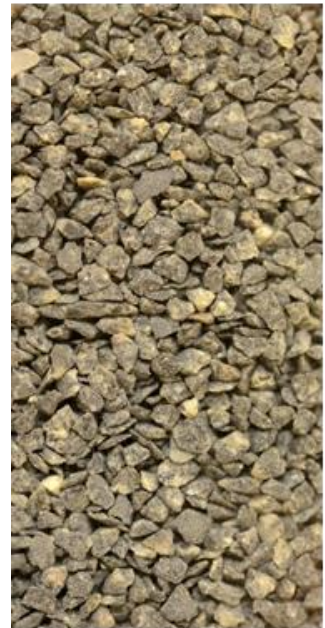

(a)



(b)

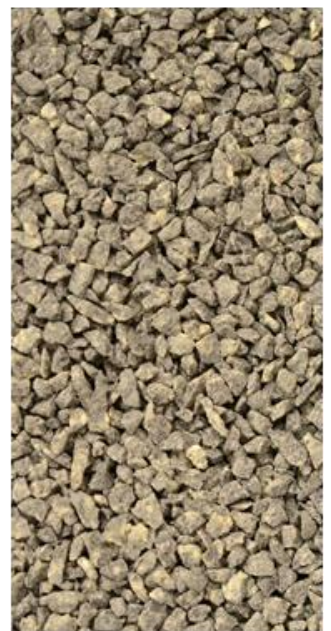

(c)

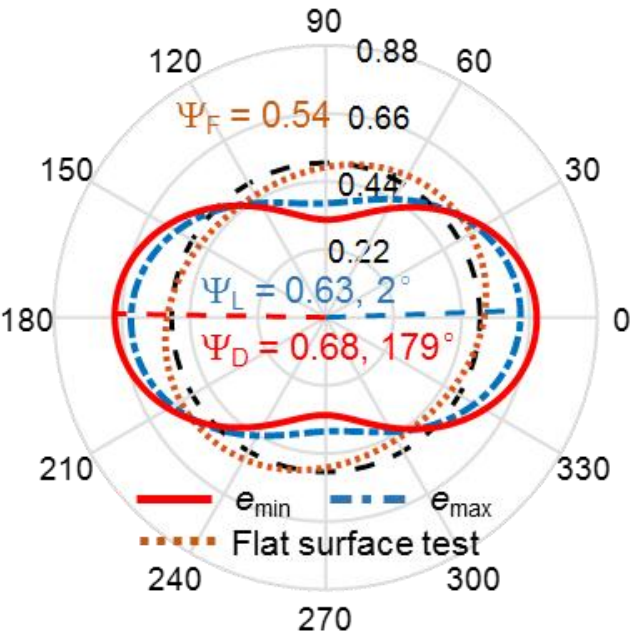

(d)

Fig. 15. Fabric anisotropy of Crushed Gabbro sand: (a) loose condition, (b) dense condition, (c) flat surface test, (d) computed ER plots.



(a)

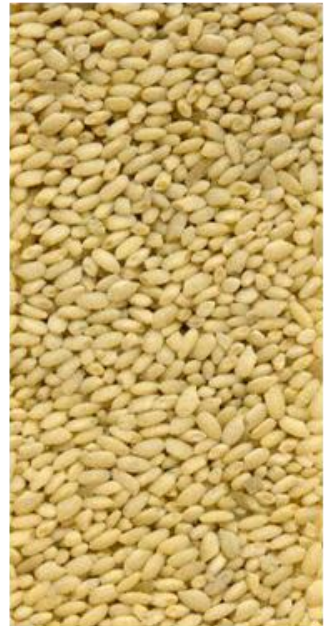

(b)

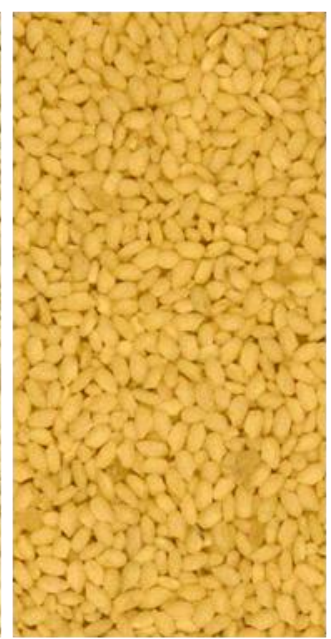

(c)

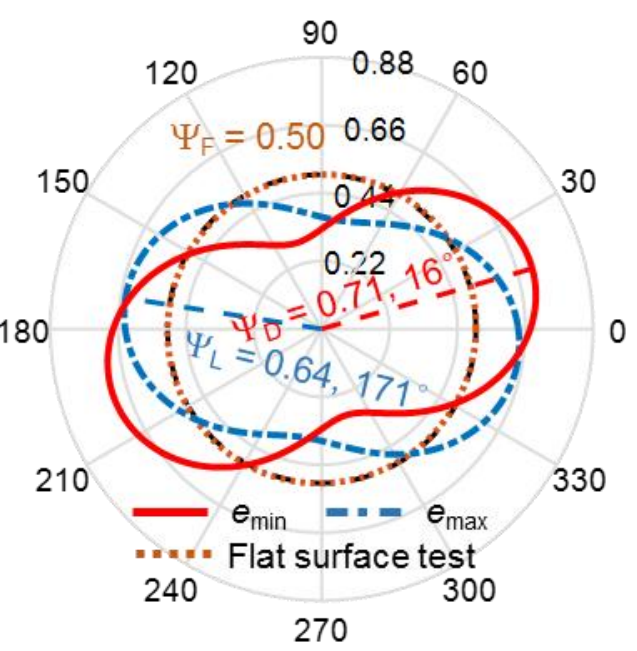

(d)

Fig. 16. Fabric anisotropy of short-grain rice: (a) loose condition, (b) dense condition, (c) flat surface test, (d) computed $E R$ plots. 


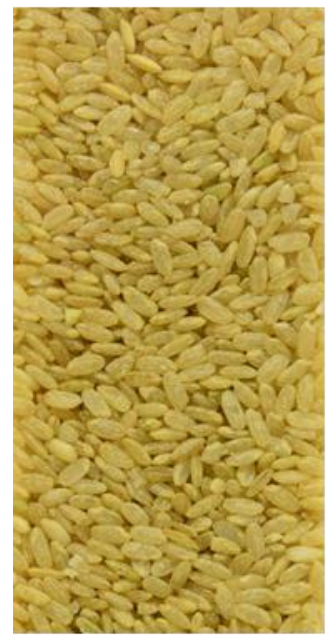

(a)

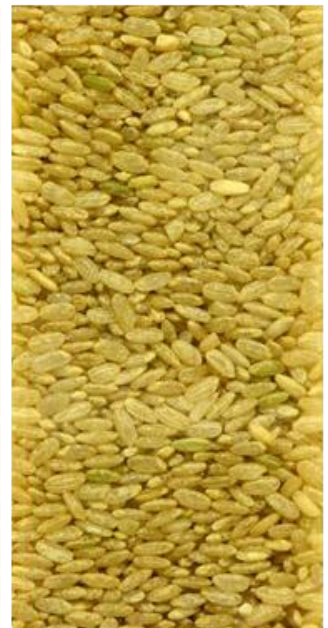

(b)

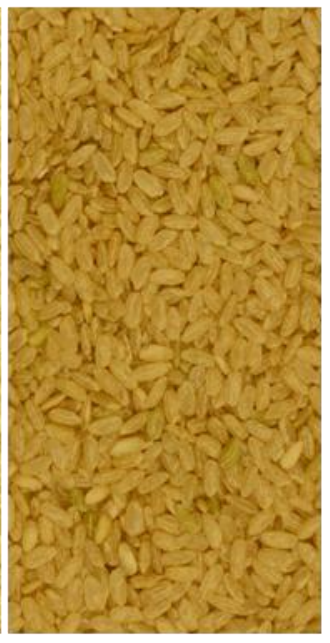

(c)

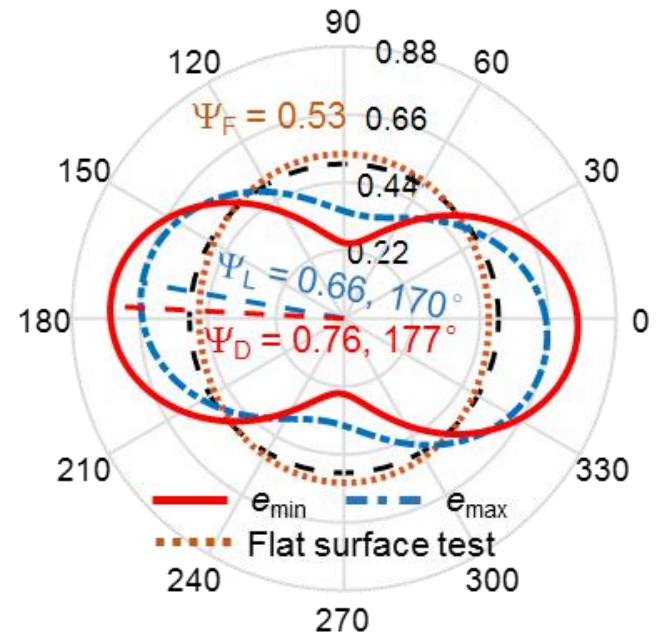

(d)

Fig. 17. Fabric anisotropy of medium-grain rice: (a) loose condition, (b) dense condition, (c) flat surface test, (d) computed ER plots.

Table. 1. The intrinsic properties and maximum fabric anisotropy of the tested materials

\begin{tabular}{|l|l|l|l|l|l|l|l|l|}
\hline Soils & $D_{50}$ & $C_{\mathrm{u}}$ & $e_{\max }$ & $e_{\min }$ & $A R$ & $\Psi_{\mathrm{L}}$ & $\Psi_{\mathrm{D}}$ & $\Psi_{\mathrm{F}}$ \\
\hline Ottawa 20 - 30 & 0.71 & 1.4 & 0.74 & 0.49 & 1.23 & 0.57 & 0.58 & 0.51 \\
\hline Michigan Dunes & 0.30 & 1.5 & 0.85 & 0.56 & 1.32 & 0.58 & 0.60 & 0.52 \\
\hline Treasure Island, California & 0.25 & 1.8 & 0.84 & 0.56 & 1.39 & 0.59 & 0.61 & 0.53 \\
\hline Oakland County, Michigan & 0.31 & 1.6 & 0.86 & 0.53 & 1.45 & 0.58 & 0.61 & 0.51 \\
\hline Class IIA, Michigan & 0.21 & 1.9 & 0.86 & 0.56 & 1.45 & 0.60 & 0.62 & 0.50 \\
\hline Capitola, California & 0.33 & 1.5 & 0.94 & 0.60 & 1.39 & 0.58 & 0.60 & 0.53 \\
\hline New Madrid, Missouri & 0.32 & 2.2 & 0.81 & 0.52 & 1.47 & 0.61 & 0.63 & 0.52 \\
\hline Chesterton, Indiana beach & 0.64 & 1.9 & 0.85 & 0.54 & 1.40 & 0.59 & 0.63 & 0.54 \\
\hline Upper Peninsula, Michigan & 0.60 & 2.8 & 0.85 & 0.54 & 1.45 & 0.60 & 0.62 & 0.54 \\
\hline Michigan 2NS & 0.50 & 2.3 & 0.82 & 0.54 & 1.49 & 0.59 & 0.62 & 0.53 \\
\hline Griffin, Indiana & 0.74 & 3.3 & 0.79 & 0.51 & 1.54 & 0.61 & 0.64 & 0.52 \\
\hline Crushed Gabbro & 0.80 & 1.5 & 0.98 & 0.60 & 1.78 & 0.63 & 0.68 & 0.54 \\
\hline Short - grain rice & 1.91 & 1.1 & 0.97 & 0.65 & 1.82 & 0.64 & 0.71 & 0.50 \\
\hline Medium - grain rice & 1.62 & 1.1 & 1.01 & 0.72 & 2.15 & 0.66 & 0.76 & 0.53 \\
\hline Long -grain rice & 1.51 & 1.1 & 1.08 & 0.85 & 3.00 & 0.70 & 0.86 & 0.51 \\
\hline
\end{tabular}

Note: $C_{\mathrm{u}}=$ coefficient of uniformity; $D_{50}=50 \%$ size $(\mathrm{mm}) ; e_{\max }$ and $e_{\min }=$ maximum and minimum index void ratios; NS = natural sand; $A R=$ aspect ratio. 
The relationships between $A R$ and $\Psi_{\mathrm{L}}, \Psi_{\mathrm{D}}$, and $\Psi_{\mathrm{F}}$ are shown in Fig. 18. Excluding the rightmost three data points which are from the rice specimens, most of the data points are concentrated in the narrow range of $1.2<A R<1.8$. As expected, all of the $\Psi_{\mathrm{F}}$ values are below 0.55 and are independent of the $A R s$. Both $\Psi_{L}$ and $\Psi_{D}$ values increase with increasing $A R$ reflecting the stronger fabric anisotropy developed in soils with more elongated particles. The relative density will affect the orientation distribution of particle long axes. Following shakedown to their dense states, soil particles display stronger preferred orientations and therefore stronger fabric anisotropy. Therefore, the $\Psi_{\mathrm{D}}$ values are larger than $\Psi_{\mathrm{L}}$ values in Fig. 18.

The $\Psi_{\mathrm{L}}$ and $\Psi_{\mathrm{D}}$ values establish the degree of cross-anisotropic fabric in soils at $e_{\max }$ and $e_{\min }$ states respectively. The relationships can be described by:

$$
\begin{aligned}
& \Psi_{D}=0.33 \ln (A R)+0.50 \\
& \Psi_{L}=0.25 \ln (A R)+0.50
\end{aligned}
$$

In the above two equations, the constant " 0.50 " is the reference isotropic fabric value that would be expected for perfectly spherical particles such as glass beads. The terms " $0.33 \ln (A R)$ " and " $0.25 \ln (A R)^{\prime}$ reflect the divergence from isotropic fabric due to the particle $A R s$. Those two equations are also plotted in Figs. 18. Eq. (18) fits the $\Psi_{D}$ values very well while Eq. (19) fits the $\Psi_{L}$ values well when $A R<1.8$ but overpredicts $\Psi_{\mathrm{D}}$ when $A R>1.8$. However, as mentioned before, the average $A R$ values for the majority of natural sands are below 1.8. Therefore, Eq. (19) should be valid for most sands.

The difference between Eqs. (18) and (19) is $0.08 \ln (A R)$ indicating how much the range of $\Psi$ depends on $A R$. Elongated soils tend to exhibit a wider range of $\Psi$. The $\Psi$ value at any relative density $D_{\mathrm{r}}=\left(e_{\max }-\right.$ $e) /\left(e_{\max }-e_{\min }\right)$ can be computed by linear interpolation between Eqs. (18) and (19): 


$$
\Psi=D_{r}\left(\Psi_{D}-\Psi_{L}\right)+\Psi_{L}=\left(0.08 D_{r}+0.25\right) \ln (A R)+0.5
$$

Although users can always determine $\Psi$ values through the RHWT presented in this study, Eq. (20) provides an alternative way to estimate $\Psi$ if the $D_{\mathrm{r}}$ and $A R$ of a soil are known.

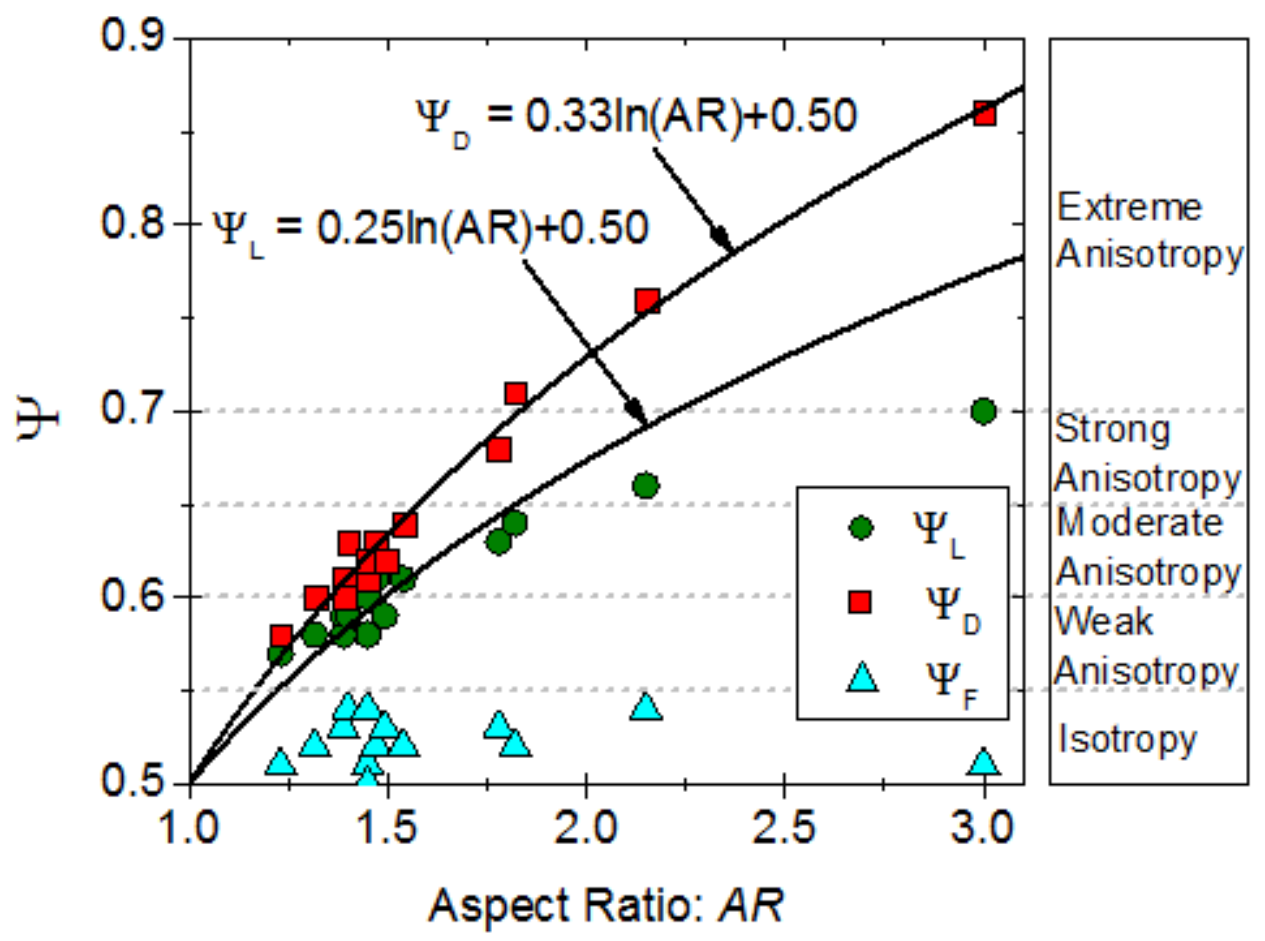

Fig. 18. The relationship between aspect ratio and degree of fabric anisotropy.

The degrees of fabric anisotropy may be divided into five levels based on $\Psi$ values. The $\Psi$ values of flat surface tests are below 0.55 , so $\Psi=[0.50,0.55]$ is classified as an isotropic fabric. For $\Psi$ values larger than 0.55 , the degrees of fabric anisotropy may be divided into four additional levels: weak anisotropy $(0.55<\Psi \leq 0.60)$, moderate anisotropy $(0.60<\Psi \leq 0.65)$, strong anisotropy $(0.65<\Psi \leq 0.70)$, and extreme anisotropy $(0.70<\Psi)$ as shown in Fig. 18.

\section{Fabric of Clay and Rock by RHWT}


The Rotational Haar Wavelet Transform technique is not limited to analysis of sand fabric. It can analyze the fabric of any material that manifests spatial grayscale changes in an image. Images can be captured by off-the-shelf cameras, cellphone cameras, digital microscopes, scanning electron microscopes, airborne drones, satellite cameras and other devices. The developed method can be used in many disciplines in which fabric analysis is needed including material science, soil science, geology, powder technology, pavement engineering agriculture, medicine and many others. Building on this paper's theme of earth materials, example analyses of clay and rock fabric will be presented in this section.

Stone et al. [65] captured a series of kaolinite clay images using an LEO (Zeiss) 1550 field emission scanning electron microscope (FESEM). The kaolinite has a size distribution of $97.5 \%$ finer than the No. 200 sieve, $68 \%$ finer than 2 microns, a liquid limit of 76 and a plastic limit of 33 . The specimen was compacted at optimum moisture content in a standard Proctor test, then freeze dried and fractured. The fracture surface was sputter coated with iridium for imaging. The clay particles on the fractured surface should be distributed somewhat randomly as they were in the flat surface tests on sands. As such, the fabric should be isotropic. Figs. 19(a) and (c) present two images of the same fracture surface at different magnifications. The RHWT method was used to evaluate the fabric anisotropy in both images. The computed $\Psi$ values were 0.54 and 0.52 respectively indicating an isotropic fabric as shown in Figs. 19(b) and (d).

In another group of tests, the clay was compacted at optimum moisture then failed in unconfined compression. After freeze drying and sputter coating with iridium, an image was taken of the failure surface. During shearing, the particle long axes become oriented in the sliding direction generating an anisotropic fabric. Figs. 20(a) and (c) capture this failure surface at two magnifications. Based on visual observation, the sliding direction of the clay particles is about $100^{\circ}$. Fig. 20 (b) and (d) provide the $E R$ plots. The computed fabric orientations are $106^{\circ}$ and $102^{\circ}$ which agree well with visual observations. 
The computed $\Psi$ values are 0.57 and 0.62 indicating that weak to moderate anisotropy was developed as a result of shearing.
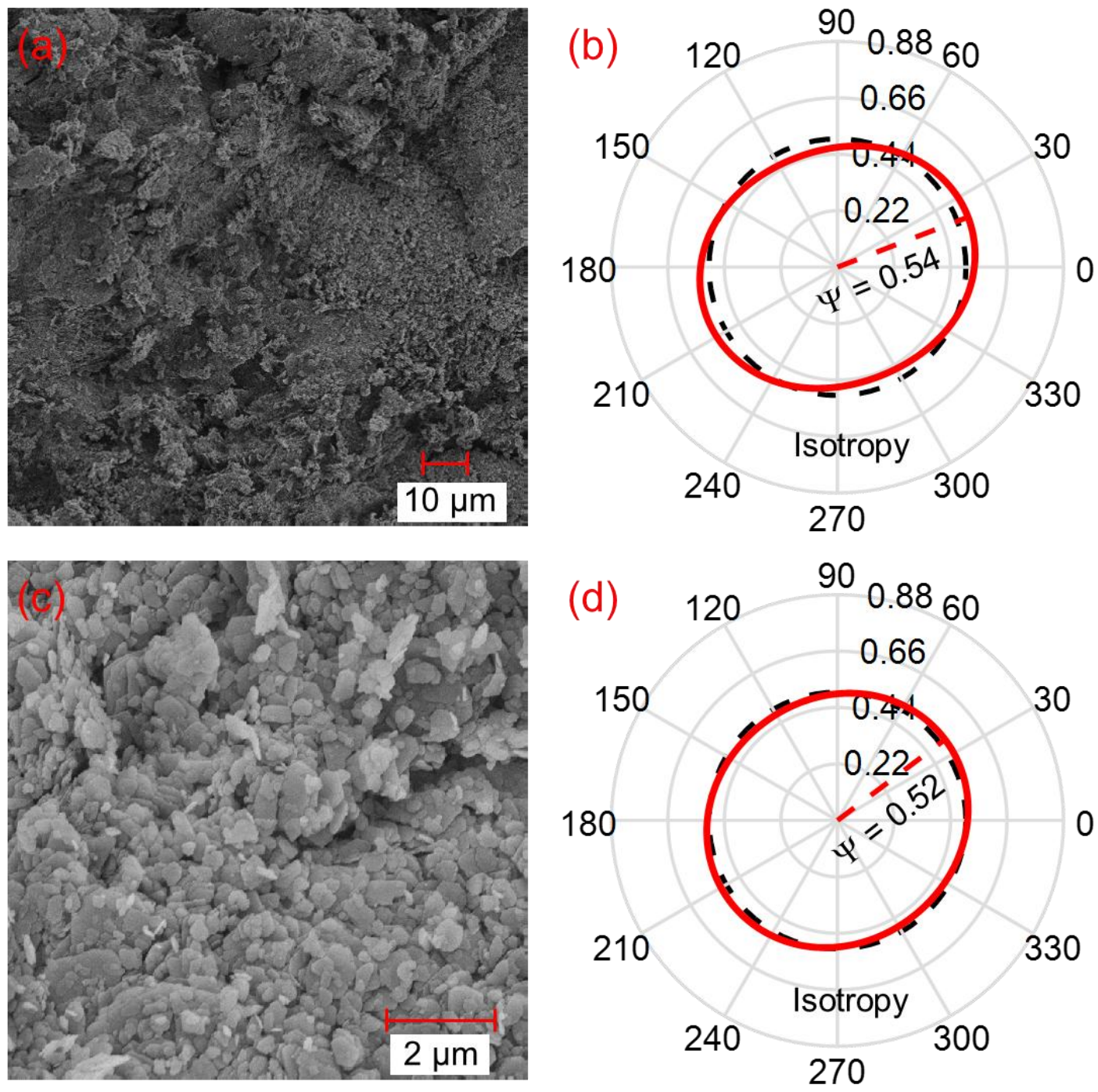

Fig. 19. Fabric anisotropy of kaolinite clay caused by fracturing. 

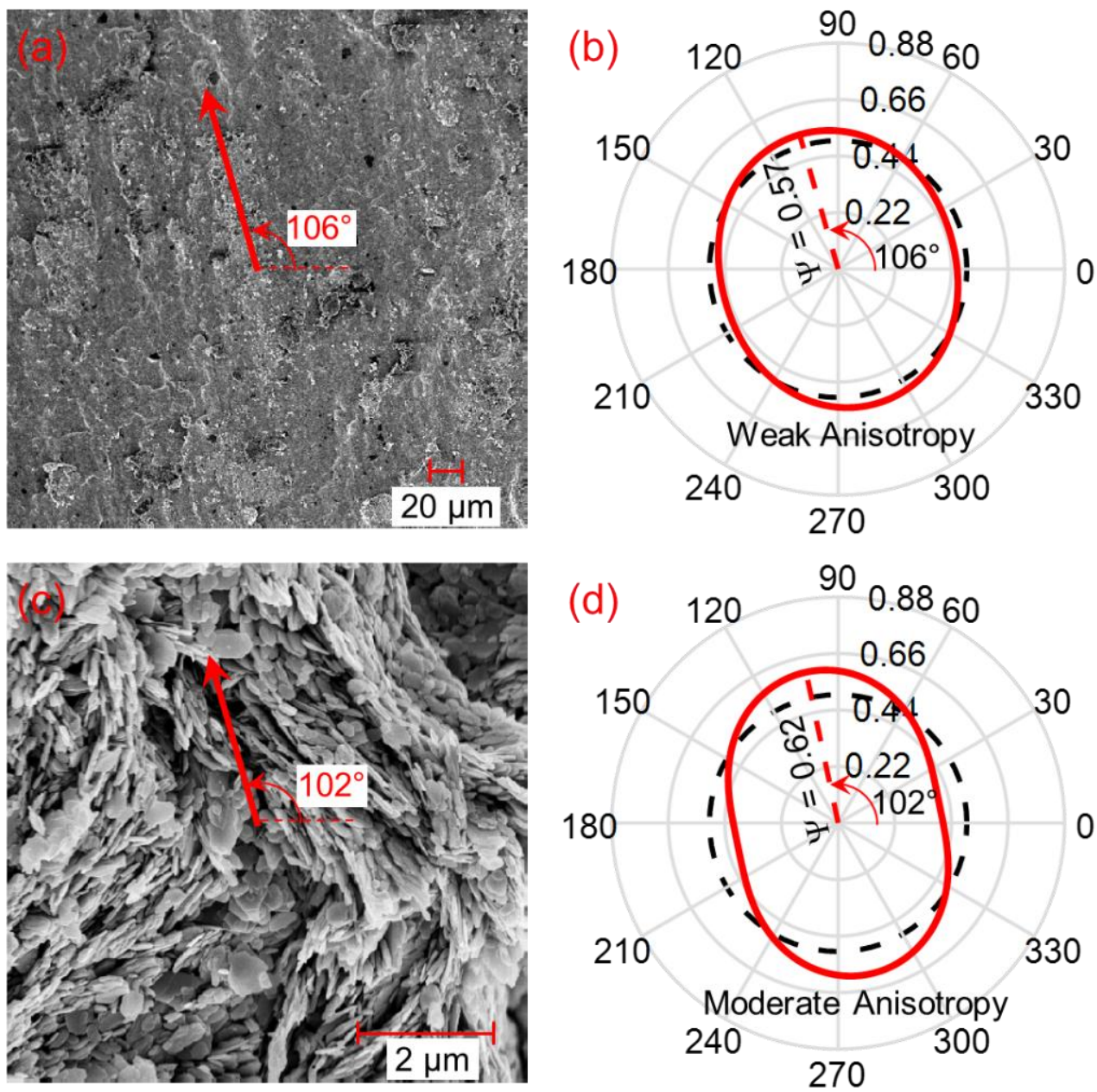

Fig. 20. Fabric anisotropy of kaolinite clay caused by shearing.

Fabric anisotropy also exists in rock. In sedimentary rocks, the fabric can arise from orientation of mineral grains during deposition. In igneous rocks, fabrics can be developed by magmatic flow orientation. In metamorphic rocks, fabrics can arise due to either deviatoric stress, or relicts inherited from their protoliths. Two rock images from the online Imperial College Rock Image Library [66] are shown in Fig. 21. Fig. 21(a) is an igneous rock showing a horizontal magmatic flow. The computed $\Psi=$ 0.65 indicates a strong anisotropic fabric. As shown in Fig. 21(b), the computed fabric orientation of $6^{\circ}$ agrees well with visual observation. Fig. 21(c) shows a metamorphic rock composed mainly of antigorite with some minor opaque hematite. The antigorite was compressed under high stress in the diagonal 
direction from upper right to lower left to form the fabric. The computational results in Fig. 21(d) show the rock has an extreme fabric with $E R_{\max }=0.73$. The computed fabric orientation of $133^{\circ}$ agrees well with visual observation.
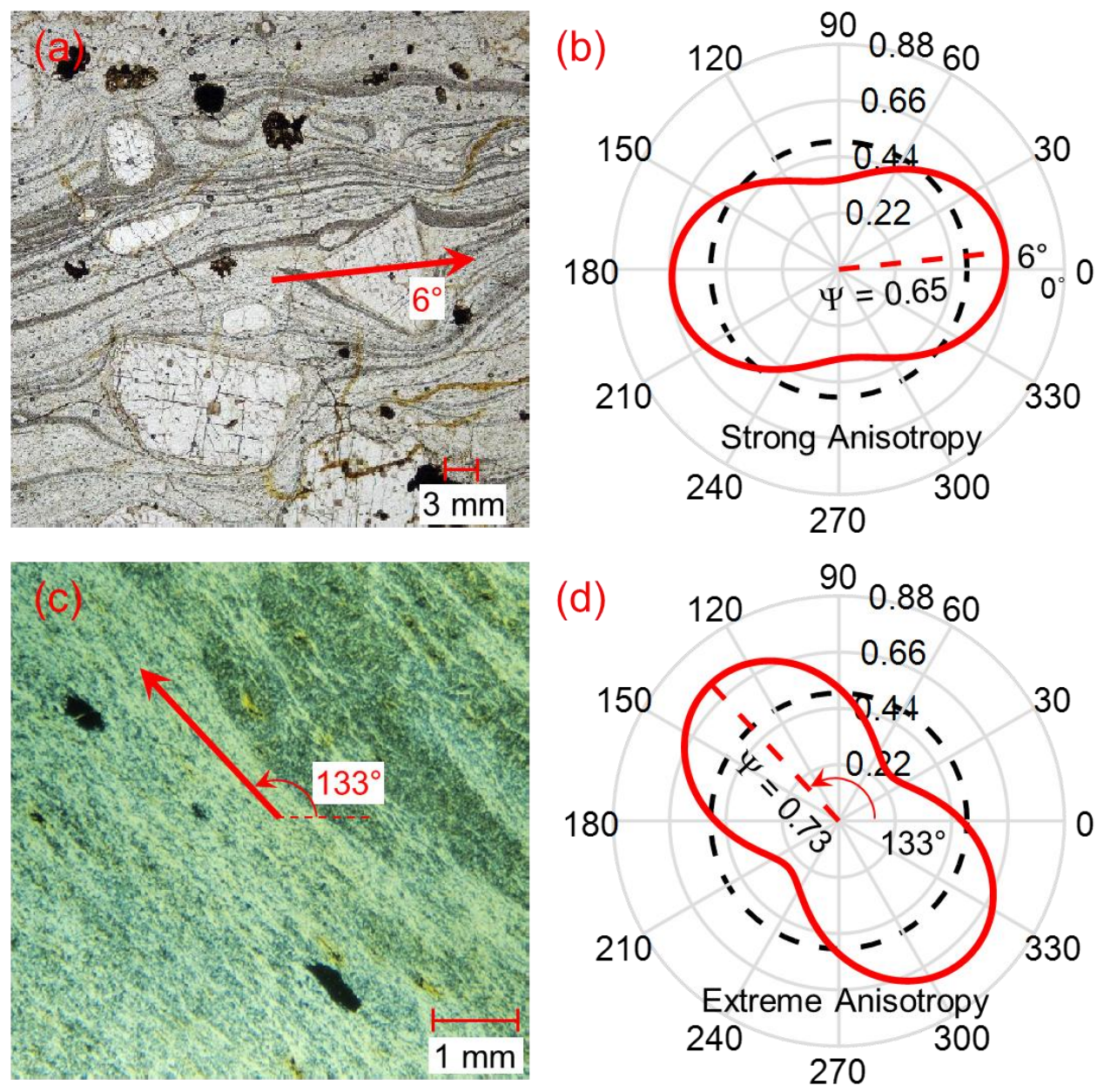

Fig. 21. Examples of fabric anisotropy in rocks.

\section{Fabric tensor from RHWT results}

In soil mechanics and constitutive modeling, the fabric of soil is usually quantified by a fabric tensor [4348, 53]:

$$
\varphi_{i j}=\int_{\Omega} P(\Omega) n_{i} n_{j} d \Omega \quad(i, j=1,2,3)
$$


where the $n_{i}(i=1,2,3)$ are the components of a unit vector $\mathbf{n}$ describing the orientation of particle long axes; the $P(\Omega)$ is a probability density function describing the distribution of $\mathbf{n} ; \Omega$ is the solid angle corresponding to the entire surface of a unit sphere; and $P(\Omega) d \Omega$ is the fraction of $\mathbf{n}$ oriented within a small solid angle $d \Omega$. The computed fabric tensor by Eq. (21) is a symmetric, third-rank matrix, which can be represented by three principal values $\varphi_{1}, \varphi_{2}$ and $\varphi_{3}$ at corresponding principal directions $\theta_{1}, \theta_{2}$ and $\theta_{3}$.

The sum of the three principal values of the fabric tensor $\varphi_{1}, \varphi_{2}$ and $\varphi_{3}$ is unity and for a crossanisotropic fabric, $\varphi_{1}$ must equal to $\varphi_{2}$. Therefore, if we know the relationship between $\varphi_{1}$ and $\varphi_{3}$, the three principal values can be computed. Such a relationship between $\varphi_{1}$ and $\varphi_{3}$ can be obtained from the RHWT of two-dimensional side images along the depositional direction such as the images in Figs. 13(a) to $17(a)$.

For a two - dimensional image, Eq. (21) can be simplified to

$$
\bar{\varphi}_{i j}=\int_{0}^{2 \pi} P(\theta) n_{i} n_{j} d \theta \quad(i, j=1,2)
$$

where $\left(n_{1}, n_{2}\right)=(\cos \theta, \sin \theta)$ and the bar indicates that it is for a 2D image. The $P(\theta)$ can be any function that satisfies:

$$
\int_{0}^{2 \pi} P(\theta) d \theta=1
$$

With a minor mathematical adjustment to satisfy Eq. (23), our Fourier idealization of the $E R$ distribution given by Eq. (14) becomes the function $P(\theta)$ :

$$
P(\theta)=\frac{2}{\pi} E R(\theta)-\frac{1}{2 \pi}=\frac{1}{2 \pi}+\frac{2 a_{2}}{\pi} \cos (2 \theta)+\frac{2 b_{2}}{\pi} \sin (2 \theta)
$$


Combining Eqs. (24) and (22), the two - dimensional fabric tensor is computed to be:

$$
\bar{\varphi}=\left[\begin{array}{cc}
0.5+a_{2} & b_{2} \\
b_{2} & 0.5-a_{2}
\end{array}\right]
$$

The $\bar{\varphi}$ is a symmetric, second-rank tensor and its two principal values $\bar{\varphi}_{1}$ and $\bar{\varphi}_{2}$ are:

$$
\left\{\begin{array}{l}
\bar{\varphi}_{1} \\
\bar{\varphi}_{3}
\end{array}\right\}=\frac{\left(\bar{\varphi}_{11}+\bar{\varphi}_{33}\right) \pm \sqrt{\left(\bar{\varphi}_{11}-\bar{\varphi}_{33}\right)^{2}+4 \bar{\varphi}_{13}^{2}}}{2}=0.5 \pm \sqrt{a_{2}^{2}+b_{2}^{2}}
$$

Comparing Eq. (26) and Eq. (15), the two principal values $\bar{\varphi}_{1}$ and $\bar{\varphi}_{3}$ are exactly $E R_{\max }$ and $E R_{\min }$. If $E R_{\max }$ and $E R_{\min }$ are replaced by $\Psi$ and $1-\Psi$ respectively, we have $\bar{\varphi}_{1}=\Psi$ and $\bar{\varphi}_{3}=1-\Psi$.

Assuming principal values $\bar{\varphi}_{1}$ and $\bar{\varphi}_{3}$ in two - dimensional images are proportional to the principal values $\varphi_{1}$ and $\varphi_{3}$ in three dimensions for cross-anisotropic soil (Oda and Nakayama 1989):

$$
\frac{\varphi_{1}}{\varphi_{3}}=\frac{\bar{\varphi}_{1}}{\bar{\varphi}_{3}}=\frac{\Psi}{1-\Psi}
$$

Since $\varphi_{1}=\varphi_{2}$ and $\varphi_{1}+\varphi_{2}+\varphi_{3}=1$, the three principal values of the fabric tensor $\varphi_{1}, \varphi_{2}$ and $\varphi_{3}$ can be obtained. The complete fabric tensor defined by Eq. (21) can finally be rewritten as:

$$
\varphi=\left[\begin{array}{ccc}
\varphi_{1} & 0 & 0 \\
0 & \varphi_{2} & 0 \\
0 & 0 & \varphi_{3}
\end{array}\right]=\frac{1}{1+\Psi}\left[\begin{array}{ccc}
\Psi & 0 & 0 \\
0 & \Psi & 0 \\
0 & 0 & 1-\Psi
\end{array}\right]
$$

The $\Psi$ can be determined using either the RHWT technique detailed in this paper or estimated by Eq. (20) with known values of $A R$ and $D_{\mathrm{r}}$. Equation (28) can be readily used in development of constitutive soil models and statistically correlated to various anisotropic soil properties. Fig. 22 plots $\varphi_{1}, \varphi_{2}$, and $\varphi_{3}$ versus $\Psi$ in which the $\varphi_{1}$ and $\varphi_{2}$ curves overlap. For isotropic fabric, $\Psi=0.5$ and therefore, $\varphi_{1}=\varphi_{2}=\varphi_{3}$ 
$=1 / 3$. For complete anisotropy, $\Psi=1.0$ and then $\varphi_{1}=\varphi_{2}=0.5$ and $\varphi_{3}=0$. As $\Psi$ increases from 0.5 to 1 , the $\varphi_{1}$ and $\varphi_{2}$ increase from $1 / 3$ to 0.5 while $\varphi_{3}$ decreases from $1 / 3$ to 0 .

The degree of cross-anisotropic fabric can also be quantified by a deviatoric fabric parameter defined by Thornton [67] as $\varphi_{d}=\varphi_{1}-\varphi_{3}$. The evolution of $\varphi_{d}$ in the shearing process has been extensively used in DEM modeling by Batteto and O'Sullivan [68] and Huang et al. [69]. Fig. 22 also plots the relationship between $\varphi_{\mathrm{d}}$ and $\Psi$. As $\Psi$ increases from 0.5 to $1, \varphi_{\mathrm{d}}$ increases from 0 to 0.5 .

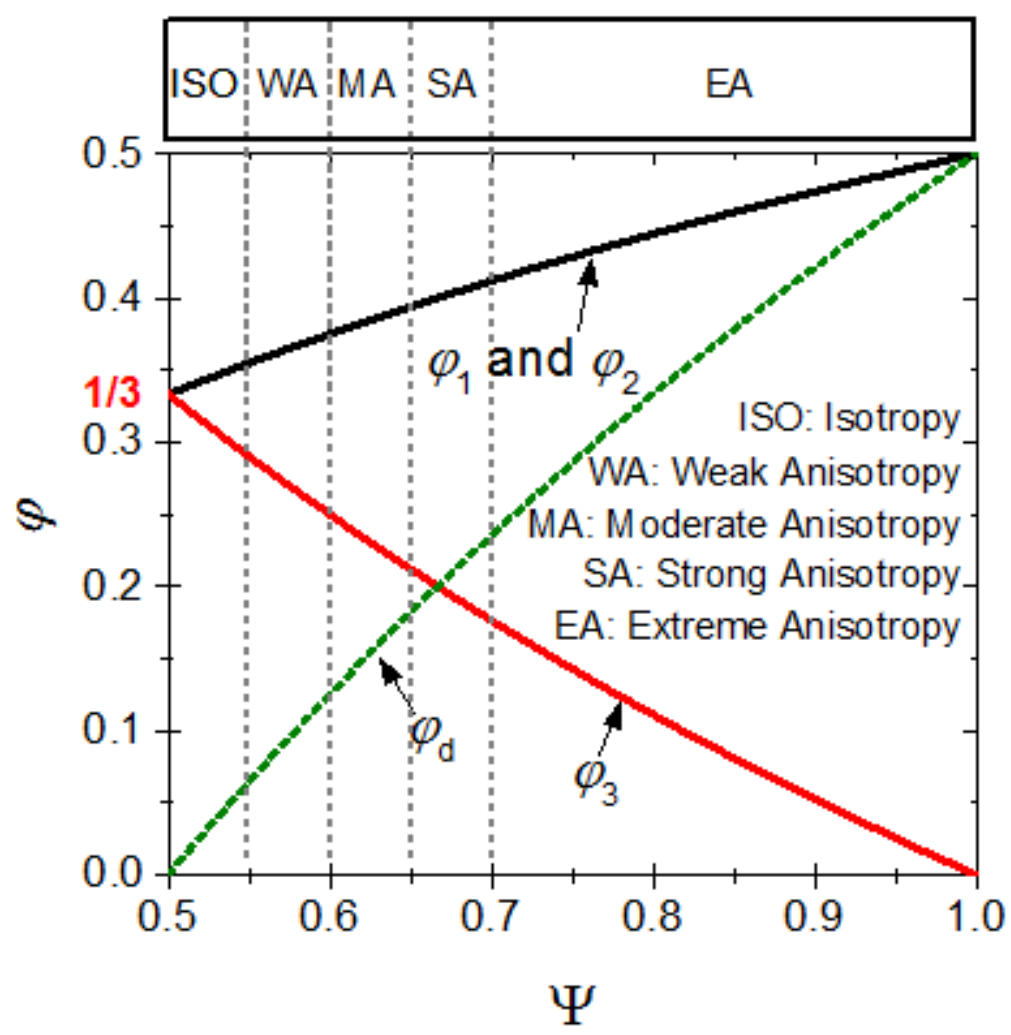

Fig. 22. Relationship between the fabric tensor and $\Psi$.

\section{Conclusions}

A rotational Haar wavelet transform (RHWT) method has been developed and implemented to characterize the fabric of particulate materials from two-dimensional images. To demonstrate the RHWT, 
the method was first used on synthetic purely anisotropic and purely isotropic grayscale images. The Energy of the Haar wavelet was shown to be minimum in the fabric direction and maximum in the direction normal to the fabric direction. An Energy Ratio, ER was defined that ranges from 0 to 1.0 and is maximum in the fabric direction. This $E R$ is plotted in a traditional polar rose diagram that shows lobes elongated in the fabric direction. For a purely isotropic material the $E R$ plot is a circle having a value of 0.5 in all directions. As a material displays stronger fabric anisotropy the maximum $E R$ extends in the fabric direction. Images of long-grain rice were taken at various degrees of particle alignment to show that $E R_{\max }$ increases as rice grain alignment increases. Because of its significance, $E R_{\max }$ was given the symbol $\Psi$ which has a theoretical range of 0.5 to 1.0 .

The RHWT was utilized on 12 sands of various geologic origins, particle sizes, coefficients of uniformity, index void ratios and most importantly, aspect ratios $(A R)$. For each material, specimens were prepared on a flat surface, in a square glass vessel at $e_{\max }$ and then densified to $e_{\min }$. As expected, images taken of the specimens prepared on a flat surface all exhibited isotropic fabric defined by $\Psi<0.55$. Images of the specimens taken through the vertical walls of the square vessel exhibited increasing fabric anisotropy as quantified by increased $\Psi$. The fabric anisotropy increased with $A R$. The $\Psi$ further increased with densification in the vessel from $e_{\max }$ to $e_{\min }$. A simple expression for $\Psi$ as a function of $A R$ and relative density, $D_{\mathrm{r}}$ was presented. A five-level fabric anisotropy classification system based on $\Psi$ was proposed.

The RHWT was also employed on scanning electron images of fractured and sheared clay surfaces as well as on several rock images to demonstrate the versatility of the method. Finally, it was shown that the commonly used fabric tensor can be elegantly defined for transversely isotropic materials in terms of the RHWT based value of $\Psi$. 


\section{Acknowledgements}

This material is based upon work supported by the U.S. National Science Foundation under Grant No. CMMI 1300010. ConeTec Investigations Ltd. and the ConeTec Education Foundation are acknowledged for their support to the Geotechnical Engineering Laboratories at the University of Michigan.

\section{References}

[1] Oda M, Nakayama H. Yield Function for Soil with Anisotropic Fabric. J Eng Mech. 1989;115(1):89-104.

[2] Kirkgard MM, Lade PV. Anisotropic three-dimensional behavior of a normally consolidated clay. Can Geotech J. 1993;30(5):848-58.

[3] Abelev AV, Lade PV. Characterization of Failure in Cross-Anisotropic Soils. J Eng Mech. 2004;130(5):599-606.

[4] Gao Z, Zhao J. Strain localization and fabric evolution in sand. Int J Solids Struct. 2013;50(2223):3634-48.

[5] Hansen JB, Gibson RE. Undrained Shear Strengths Of Anisotropically Consolidated Clays. Géotechnique. 1949;1(3):189-200.

[6] Mitchell JK. The fabric of natural clays and its relation to engineering properties. Proceedings of the Thirty-Fifth Annual Meeting of the Highway Research Board. Washington, D.C.: Highway Research Board, 1956. p. 673-713.

[7] Jakobsen B. Some fundamental properties of sand. Proceedings of 4th international conference on 
soil mechanics. London 1957. p. 167-71.

[8] Lo KY. Stability of Slopes in Anisotropic Soils. J Soil Mech Found Div. 1965;91(4):85-106.

[9] Duncan JM, Seed HB. Strength variation along failure surfaces in clay. J Soil Mech Found Div. 1966;92(SM6):81-104.

[10] Lo KY, Milligan V. Shear strength properties of two stratified clays. J Soil Mech Found Div. 1967;93(SM1):1-15.

[11] Saada AS. Testing of Anisotropic Clay Soils. J Soil Mech Found Div. 1970;99(5):1847-52.

[12] Lo KY, Morin JP. Strength Anisotropy and Time Effects of Two Sensitive Clays. Can Geotech J. 1972;9(3):261-77.

[13] Saada AS, Ou CD. Strain-Stress Relations and Failure of Anisotropic Clays. J Soil Mech Found Div. 1973;99(12):1091-111.

[14] Prevost JH. Anisotropic Undrained Stress-Strain behavior of clays. J Geotech Eng Div. 1978;104(8):1075-90.

[15] Yong RN, Silvestri V. Anisotropic behaviour of a sensitive clay. Can Geotech J. 1979;16(2):335-50.

[16] Graham J, Houlsby GT. Anisotropic elasticity of a natural clay. Géotechnique. 1983;33(2):165-80.

[17] Dewhurst DN, Aplin AC, Sarda J-P. Influence of clay fraction on pore-scale properties and hydraulic conductivity of experimentally compacted mudstones. J Geophys Res Solid Earth. 1999;104(B12):2926174.

[18] Kurukulasuriya LC, Oda M, Kazama H. Anisotropy of Undrained Shear Strength of an OverConsolidated Soil by Triaxial and Plane Strain Tests. Soils Found. 1999;39(1):21-9.

[19] Nishimura S, Minh NA, Jardine RJ. Shear strength anisotropy of natural London Clay. Géotechnique. 2007;57(1):49-62.

[20] Anantanasakul P, Yamamuro JA, Lade PV. Three-dimensional drained behavior of normally consolidated anisotropic kaolin clay. Soils Found. 2012;52(1):146-59. 
[21] Oda M. INITIAL FABRICS AND THEIR RELATIONS TO MECHANICAL PROPERTIES OF GRANULAR MATERIAL. Soils Found. 1972;12(1):17-36.

[22] Oda M, Koishikawa I, Higuchi T. Experimental study of anisotropic shear strength of sand by plane strain test. Soils Found. 1978;18(1):25-38.

[23] Tatsuoka F, Sakamoto M, Kawamura T, Fukushima S. Strength and deformation characteristics of sand in plane strain compression at extremely low pressures. Soils Found. 1986;26(1):65-84.

[24] Guo P. Modified Direct Shear Test for Anisotropic Strength of Sand. J Geotech Geoenviron Eng. 2008;134(9):1311-8.

[25] Tong Z, Fu P, Zhou S, Dafalias YF. Experimental investigation of shear strength of sands with inherent fabric anisotropy. Acta Geotech. 2014;9(2):257-75.

[26] Oda M. Anisotropic Strength of Cohesionless Sands. Journal of the Geotechnical Engineering Division. 1981;107(9):1219-31.

[27] Azami A, Pietruszczak S, Guo P. Bearing capacity of shallow foundations in transversely isotropic granular media. Int J Numer Anal Meth Geomech. 2009;34(8):771-93.

[28] Arthur JRF, Menzies BK. Inherent anisotropy in a sand. Géotechnique. 1972;22(1):115-28.

[29] Arthur JRF, Phillips AB. Homogeneous and layered sand in triaxial compression. Géotechnique. $1975 ; 25(4): 799-815$.

[30] Wong RKS, Arthur JRF. Induced and inherent anisotropy in sand. Géotechnique. 1985;35(4):471-81.

[31] Arthur JRF, Chua KS, Dunstan T. Induced anisotropy in a sand. Géotechnique. 1977;27(1):13-30.

[32] Lam W-K, Tatsuoka F. Effects of initial anisotropic fabric and .SIGMA.2 on strength and deformation

[33] Ochiai H, Lade PV. Three-Dimensional Behavior of Sand with Anisotropic Fabric. J Geotech Eng. 1983;109(10):1313-28.

[34] Rodriguez NM, Lade PV. Effects of Principal Stress Directions and Mean Normal Stress on Failure Criterion for Cross-Anisotropic Sand. J Eng Mech. 2013;139(11):1592-601. 
[35] Yang LT, Li X, Yu HS, Wanatowski D. A laboratory study of anisotropic geomaterials incorporating recent micromechanical understanding. Acta Geotech. 2015;11(5):1111-29.

[36] Meyerhof GG. Bearing capacity of anisotropic cohesionless soils. Can Geotech J. 1978;15(4):592-5.

[37] Oda M, Koishikawa I. Effect of strength anisotropy on bearing capacity of shallow footing in a dense sand. Soils Found. 1979;19(3):15-28.

[38] Hosseininia ES. Investigating the micromechanical evolutions within inherently anisotropic granular materials using discrete element method. Granular Matter. 2012;14(4):483-503.

[39] Ng T-T. Shear strength and micro-descriptors of bidisperse ellipsoids under different loading paths. Mechanics of Materials. 2009;41(6):748-63.

[40] Zhao J, Guo N. Unique critical state characteristics in granular media considering fabric anisotropy. Géotechnique. 2013;63(8):695-704.

[41] Fu P, Dafalias YF. Fabric evolution within shear bands of granular materials and its relation to critical state theory. Int J Numer Anal Meth Geomech. 2010;35(18):1918-48.

[42] Yimsiri S, Soga K. DEM analysis of soil fabric effects on behaviour of sand. Géotechnique. 2010;60(6):483-95.

[43] Pietruszczak S, Mroz Z. Formulation of anisotropic failure criteria incorporating a microstructure tensor. Comput Geotech. 2000;26(2):105-12.

[44] Pietruszczak S, Mroz Z. On failure criteria for anisotropic cohesive-frictional materials. Int J Numer Anal Meth Geomech. 2001;25(5):509-24.

[45] Li XS, Dafalias YF. Constitutive Modeling of Inherently Anisotropic Sand Behavior. J Geotech Geoenviron Eng. 2002;128(10):868-80.

[46] Dafalias YF, Papadimitriou AG, Li XS. Sand Plasticity Model Accounting for Inherent Fabric Anisotropy. J Eng Mech. 2004;130(11):1319-33.

[47] Lade PV. Modeling failure in cross-anisotropic frictional materials. Int J Solids Struct. 
2007;44(16):5146-62.

[48] Lade PV. Failure Criterion for Cross-Anisotropic Soils. J Geotech Geoenviron Eng. 2008;134(1):117-

24.

characteristics of sand. Soils Found. 1988;28(1):89-106.

[49] Schweiger HF, Wiltafsky C, Scharinger F, Galavi V. A multilaminate framework for modelling induced and inherent anisotropy of soils. Géotechnique. 2009;59(2):87-101.

[50] Liu MD, Indraratna BN. General Strength Criterion for Geomaterials Including Anisotropic Effect. Int J Geomech. 2011;11(3):251-62.

[51] Yao Y-P, Kong Y-X. Extended UH Model: Three-Dimensional Unified Hardening Model for Anisotropic Clays. J Eng Mech. 2012;138(7):853-66.

[52] Kong Y, Zhao J, Yao Y. A failure criterion for cross-anisotropic soils considering microstructure. Acta Geotech. 2012;8(6):665-73.

[53] Gao Z, Zhao J. Efficient Approach to Characterize Strength Anisotropy in Soils. J Eng Mech. 2012;138(12):1447-56.

[54] Fonseca J, O'Sullivan C, Coop MR, Lee PD. Quantifying the evolution of soil fabric during shearing using directional parameters. Géotechnique. 2013;63(6):487-99.

[55] Fonseca J, O'Sullivan C, Coop MR, Lee PD. Quantifying the evolution of soil fabric during shearing using scalar parameters. Géotechnique. 2013;63(10):818-29.

[56] Yang ZX, Li XS, Yang J. Quantifying and modelling fabric anisotropy of granular soils. Géotechnique. 2008;58(4):237-48.

[57] Shin S, Hryciw RD. Wavelet Analysis of Soil Mass Images for Particle Size Determination. J Comput Civil Eng. 2004;18(1):19-27.

[58] Hryciw RD, Ohm H-S, Zhou J. Theoretical Basis for Optical Granulometry by Wavelet Transformation. J Comput Civil Eng. 2015;29(3):04014050. 
[59] Ohm H-S, Hryciw RD. Size Distribution of Coarse-Grained Soil by Sedimaging. J Geotech Geoenviron Eng. 2014;140(4):04013053.

[60] Chandan C, Sivakumar K, Masad E, Fletcher T. Application of Imaging Techniques to Geometry Analysis of Aggregate Particles. J Comput Civil Eng. 2004;18(1):75-82.

[61] Zheng J, Hryciw RD. Traditional soil particle sphericity, roundness and surface roughness by computational geometry. Géotechnique. 2015;65(6):494-506.

[62] ASTM. (2014a). Standard test methods for maximum index density and unit weight of soils using a vibratory table. ASTM D4253-14, West Conshohocken, PA.

[63] ASTM. (2014b). Standard test methods for minimum index density and unit weight of soils and calculation of relative density. ASTM D4254-14, West Conshohocken, PA.

[64] Zheng J, Hryciw RD. Index Void Ratios of Sands from Their Intrinsic Properties. J Geotech Geoenviron Eng. 2016;142(12):1-10.

[65] Stone G, Dove JE, Han N, Dove P. Clay Mineral Image Collection for Education in Geotechnical Engineering and the Earth Sciences. 2015, http://dx.doi.org/10.17632/xwj98nb39r.1.

[66] Imperial College Rock Image Library, https://wwwf.imperial.ac.uk/earthscienceandengineering/rocklibrary/; 2016 [accessed 1.5.2016].

[67] Thornton, C. Numerical simulations of deviatoric shear deformation of granular media. Géotechnique 2000; 50(1), 43-53.

[68] Barreto D, O'Sullivan C. The influence of inter-particle friction and the intermediate stress ratio on soil response under generalised stress conditions. Granular Matter. 2012;14(4):505-21.

[69] Huang X, Hanley KJ, O'Sullivan C, Kwok CY. Exploring the influence of interparticle friction on critical state behaviour using DEM. Int J Numer Anal Meth Geomech. 2014;38(12):1276-97. 
Table. 1. The intrinsic properties and maximum fabric anisotropy of the tested materials

\begin{tabular}{|l|l|l|l|l|l|l|l|l|}
\hline Soils & $D_{50}$ & $C_{\mathrm{u}}$ & $e_{\max }$ & $e_{\min }$ & $A R$ & $\Psi_{\mathrm{L}}$ & $\Psi_{\mathrm{D}}$ & $\Psi_{\mathrm{F}}$ \\
\hline Ottawa 20-30 & 0.71 & 1.4 & 0.74 & 0.49 & 1.23 & 0.57 & 0.58 & 0.51 \\
\hline Michigan Dunes & 0.30 & 1.5 & 0.85 & 0.56 & 1.32 & 0.58 & 0.60 & 0.52 \\
\hline Treasure Island, California & 0.25 & 1.8 & 0.84 & 0.56 & 1.39 & 0.59 & 0.61 & 0.53 \\
\hline Oakland County, Michigan & 0.31 & 1.6 & 0.86 & 0.53 & 1.45 & 0.58 & 0.61 & 0.51 \\
\hline Class IIA, Michigan & 0.21 & 1.9 & 0.86 & 0.56 & 1.45 & 0.60 & 0.62 & 0.50 \\
\hline Capitola, California & 0.33 & 1.5 & 0.94 & 0.60 & 1.39 & 0.58 & 0.60 & 0.53 \\
\hline New Madrid, Missouri & 0.32 & 2.2 & 0.81 & 0.52 & 1.47 & 0.61 & 0.63 & 0.52 \\
\hline Chesterton, Indiana beach & 0.64 & 1.9 & 0.85 & 0.54 & 1.40 & 0.59 & 0.63 & 0.54 \\
\hline Upper Peninsula, Michigan & 0.60 & 2.8 & 0.85 & 0.54 & 1.45 & 0.60 & 0.62 & 0.54 \\
\hline Michigan 2NS & 0.50 & 2.3 & 0.82 & 0.54 & 1.49 & 0.59 & 0.62 & 0.53 \\
\hline Griffin, Indiana & 0.74 & 3.3 & 0.79 & 0.51 & 1.54 & 0.61 & 0.64 & 0.52 \\
\hline Crushed Gabbro & 0.80 & 1.5 & 0.98 & 0.60 & 1.78 & 0.63 & 0.68 & 0.54 \\
\hline Short - grain rice & 1.91 & 1.1 & 0.97 & 0.65 & 1.82 & 0.64 & 0.71 & 0.50 \\
\hline Medium - grain rice & 1.62 & 1.1 & 1.01 & 0.72 & 2.15 & 0.66 & 0.76 & 0.53 \\
\hline Long -grain rice & 1.51 & 1.1 & 1.08 & 0.85 & 3.00 & 0.70 & 0.86 & 0.51 \\
\hline
\end{tabular}

Note: $C_{\mathrm{u}}=$ coefficient of uniformity; $D_{50}=50 \%$ size $(\mathrm{mm}) ; e_{\max }$ and $e_{\min }=$ maximum and minimum index void ratios; NS = natural sand; $A R=$ aspect ratio. 


\section{Fiver}

\begin{tabular}{|ll|l|l|l|l|l|}
\hline 200 & 150 & 100 & 50 & 100 & 150 & 200 \\
\hline 200 & 150 & 100 & 50 & 100 & 150 & 200 \\
\hline 200 & 150 & 100 & 50 & 100 & 150 & 200 \\
200 & 150 & 100 & 50 & 100 & 150 & 200 \\
\hline 200 & 150 & 100 & 50 & 100 & 150 & 200 \\
\hline 200 & 150 & 100 & 50 & 100 & 150 & 200 \\
\hline 200 & 150 & 100 & 50 & 100 & 150 & 200 \\
\hline 200 & 150 & 100 & 50 & 100 & 150 & 200 \\
\hline
\end{tabular}











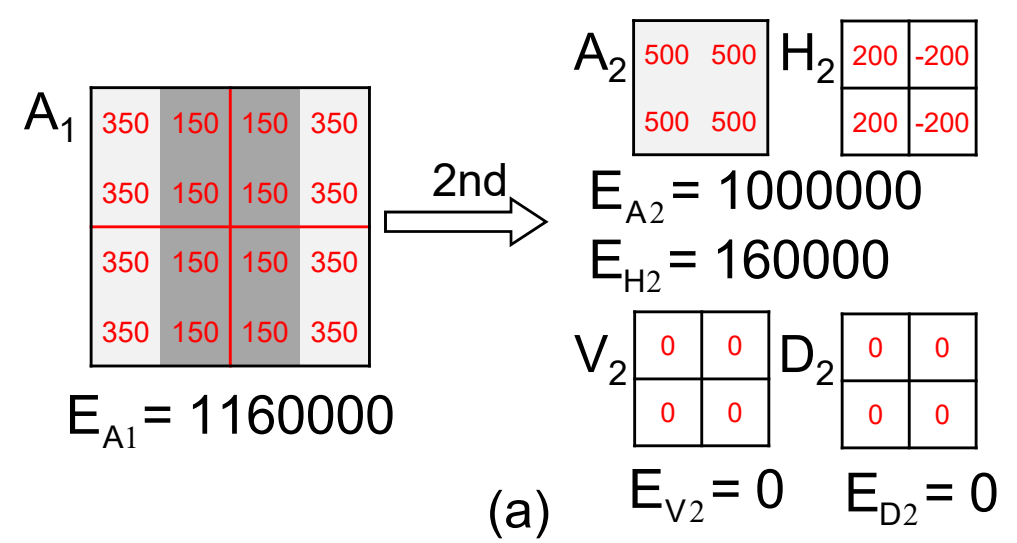

$$
\begin{aligned}
& \mathrm{A}_{3}{ }_{1000} \mathrm{H}_{3} 0
\end{aligned}
$$

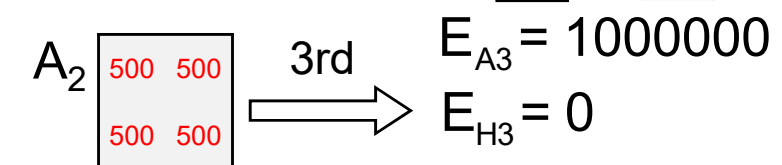

$$
\begin{aligned}
& E_{A_{2}}=1000000 \quad V_{3} \circ D_{3} 0 \\
& E_{\mathrm{V} 3}=0 \quad E_{D 3}=0
\end{aligned}
$$

(b) 


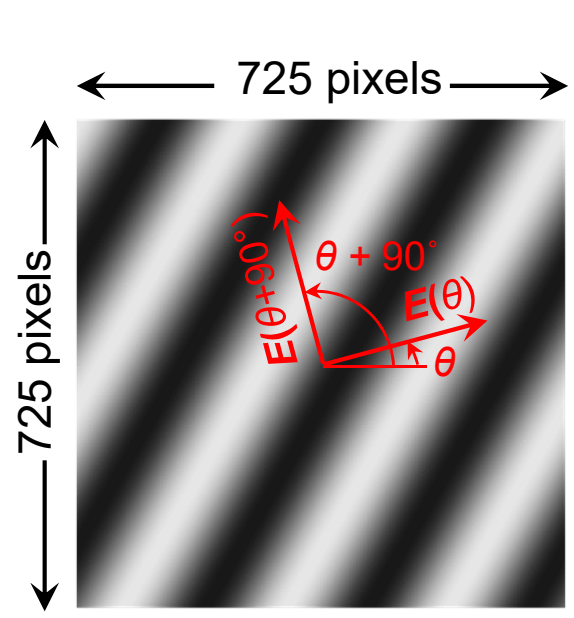

(a)

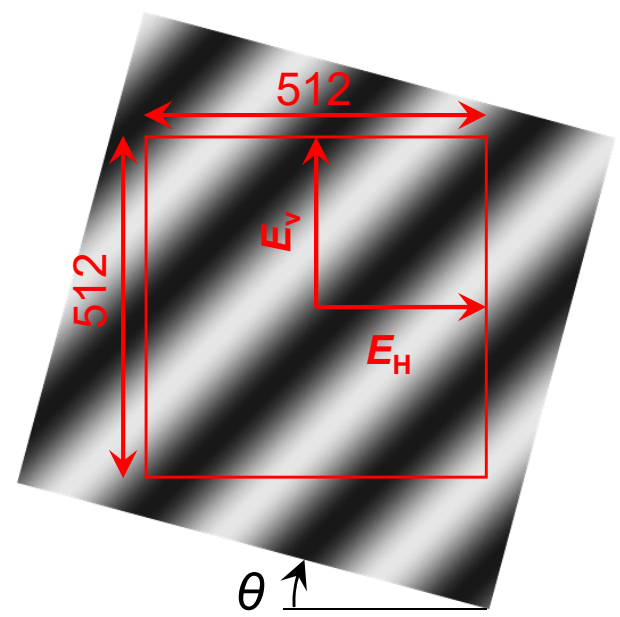

(b)

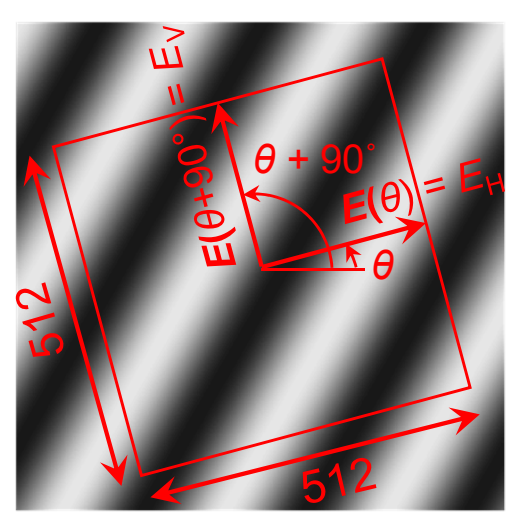

(c)

c) 


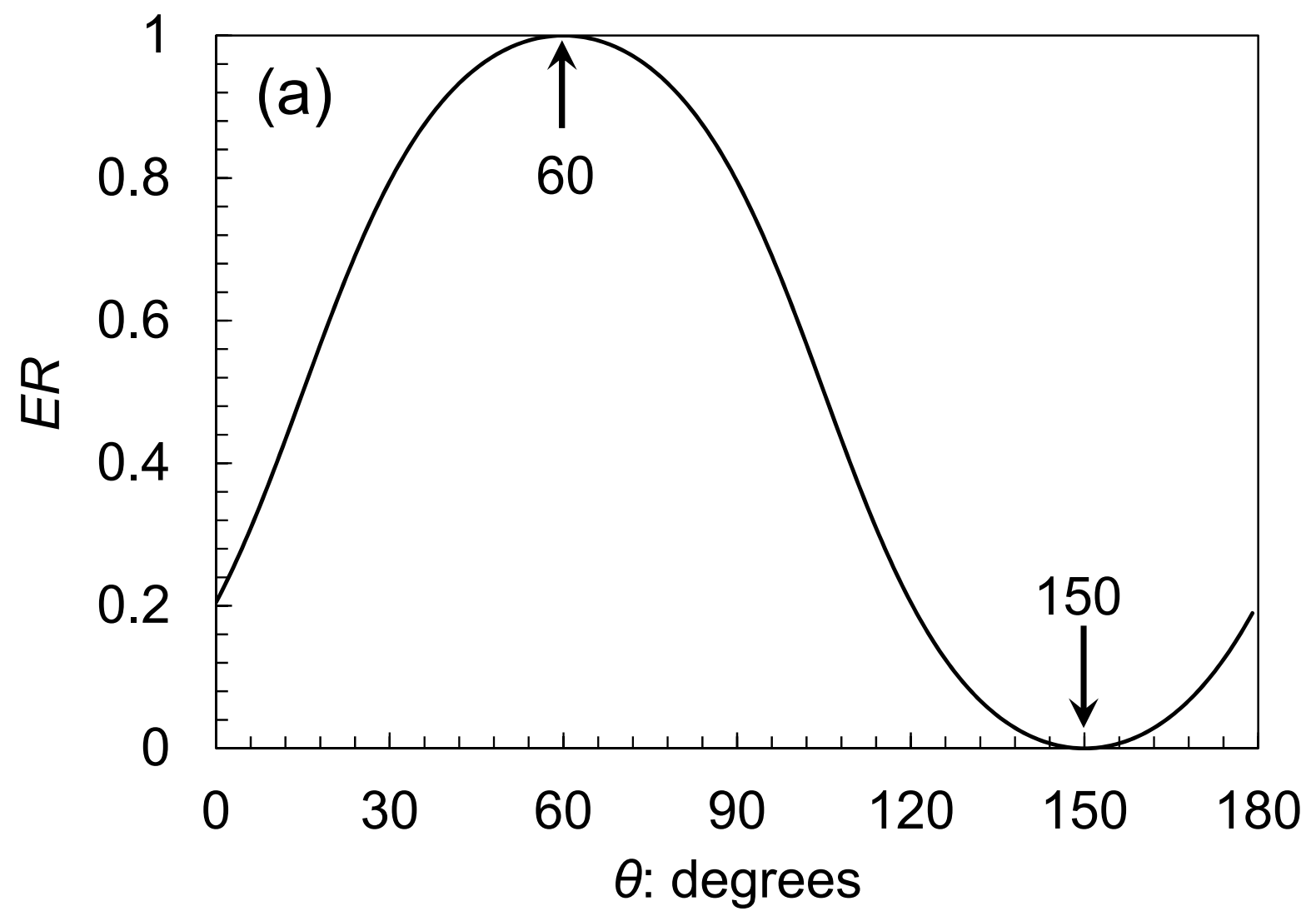

(b)

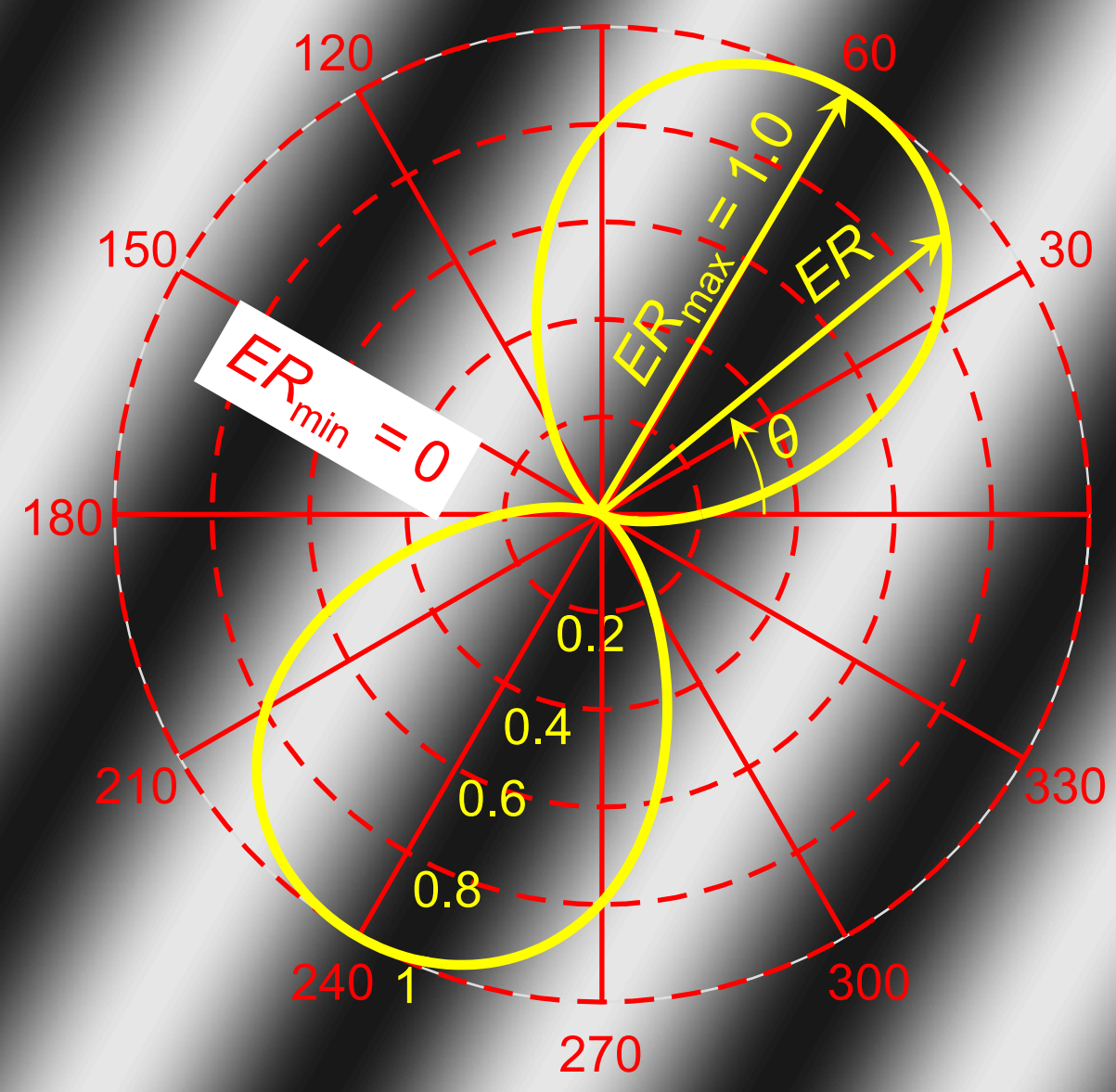




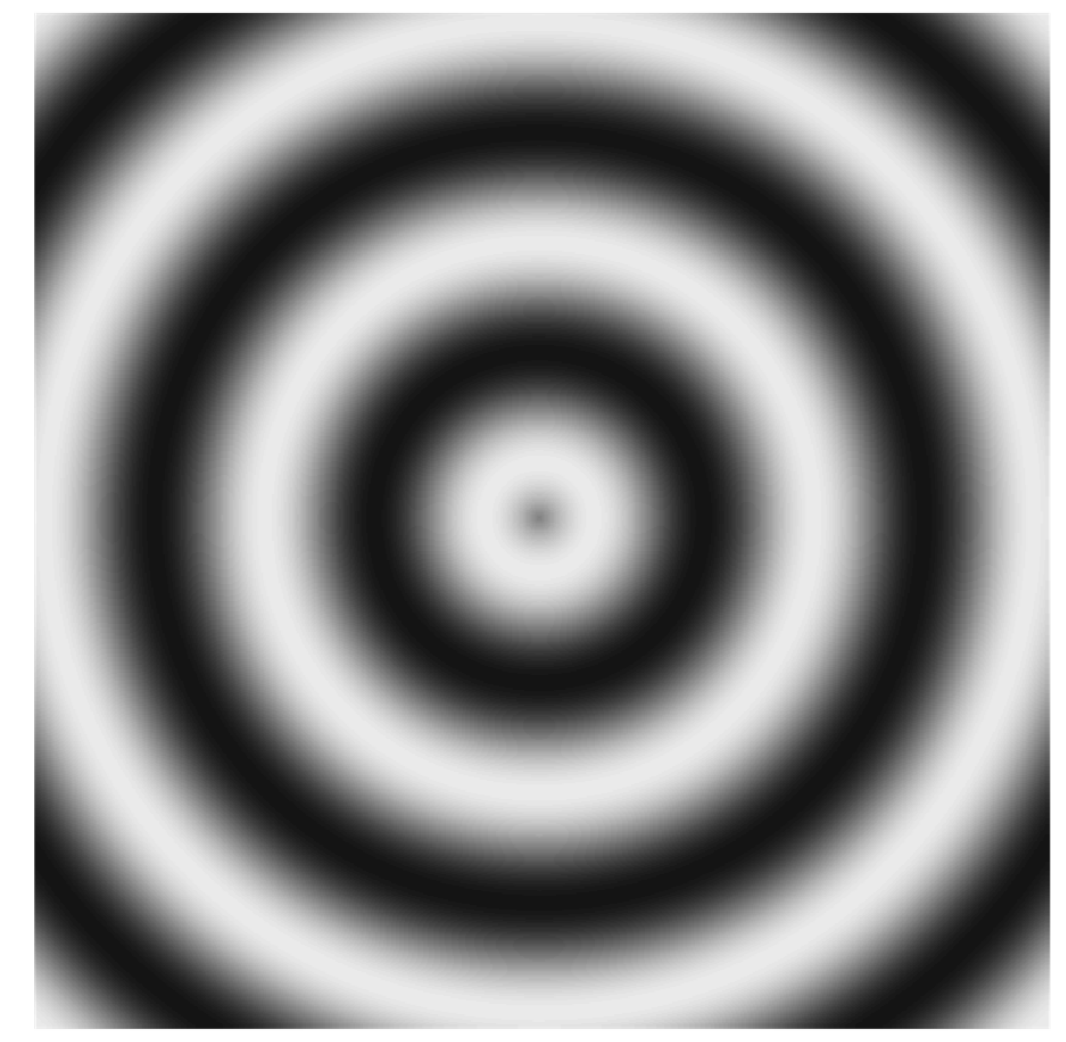

(a)

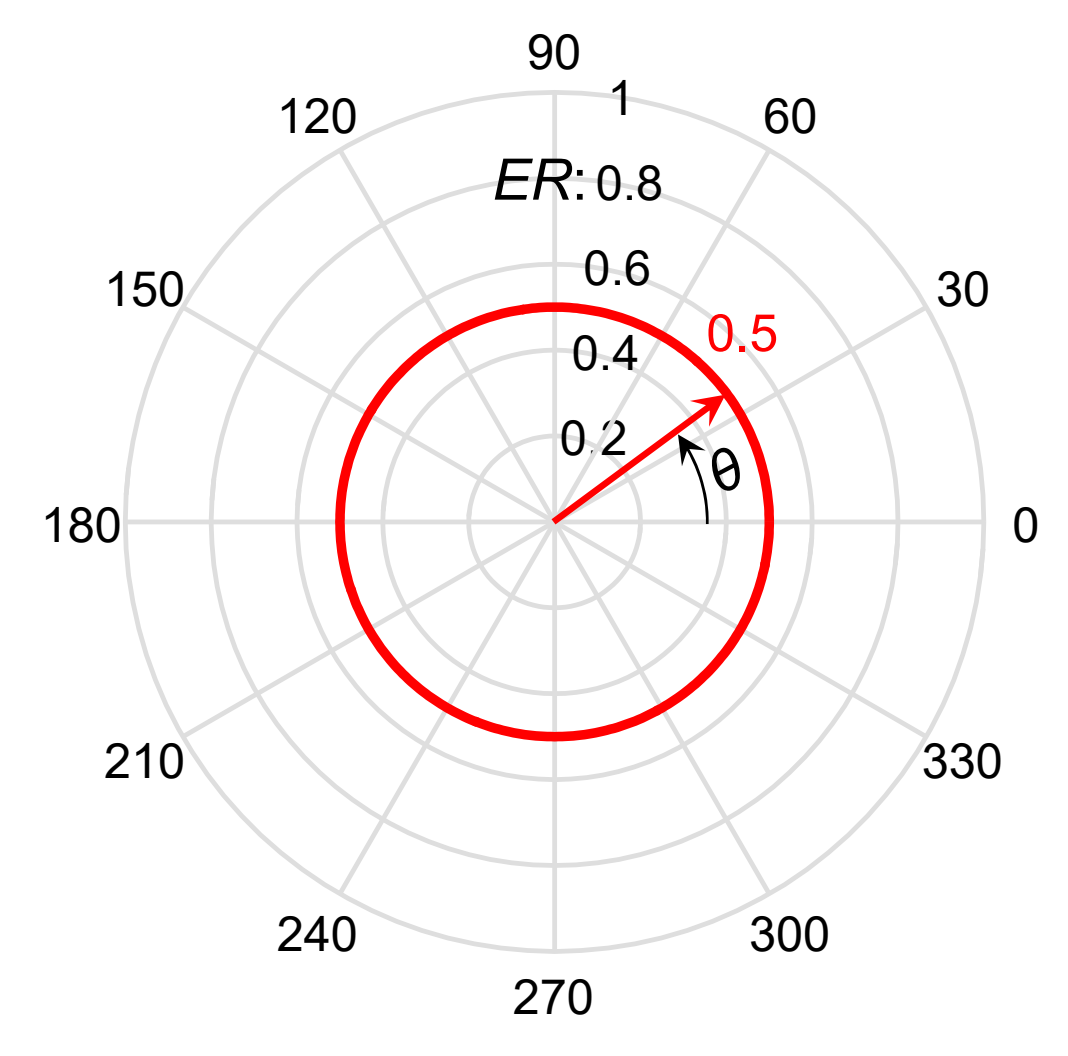

(b) 


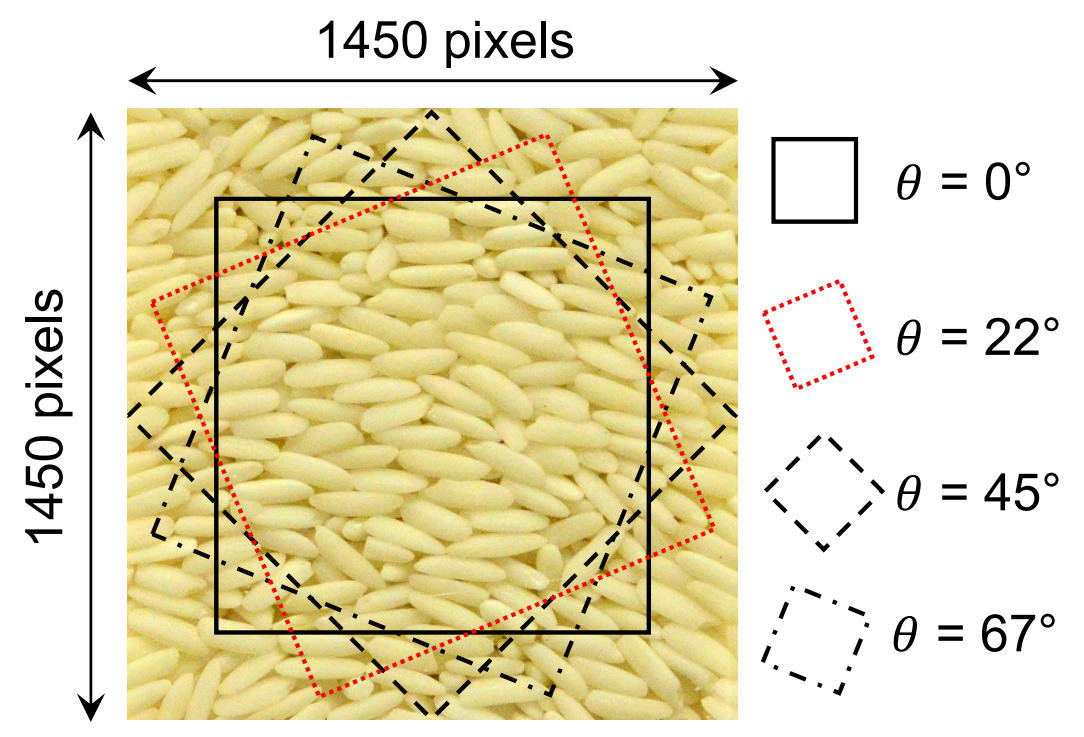





$A_{7}: 8 \times 8$

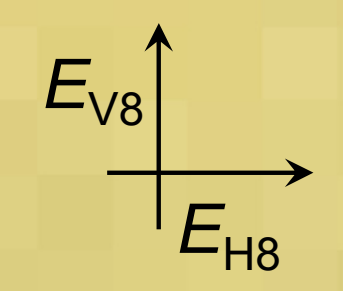




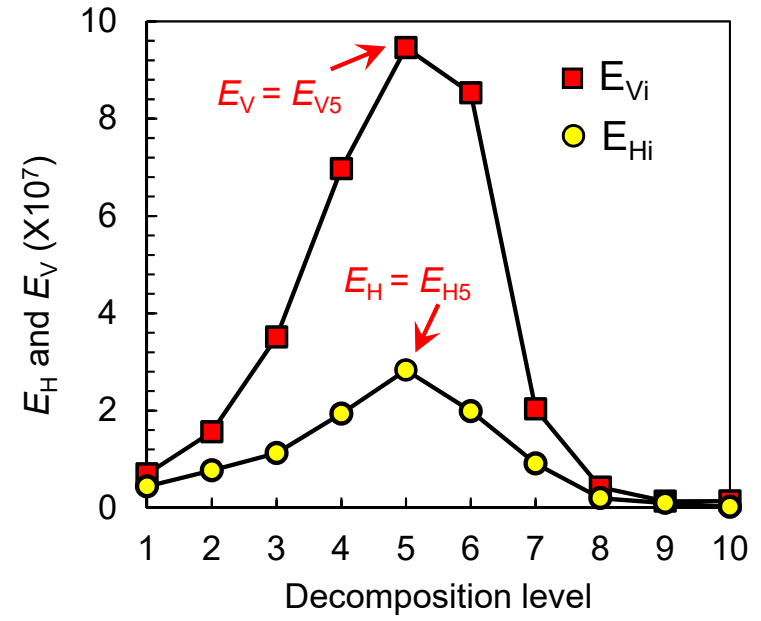




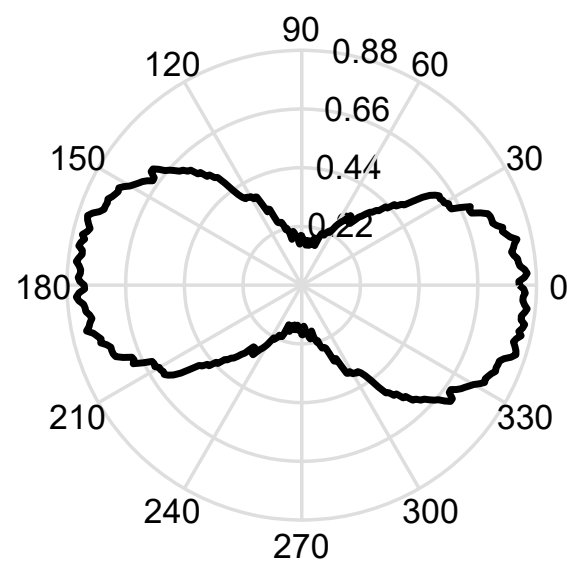

(a)

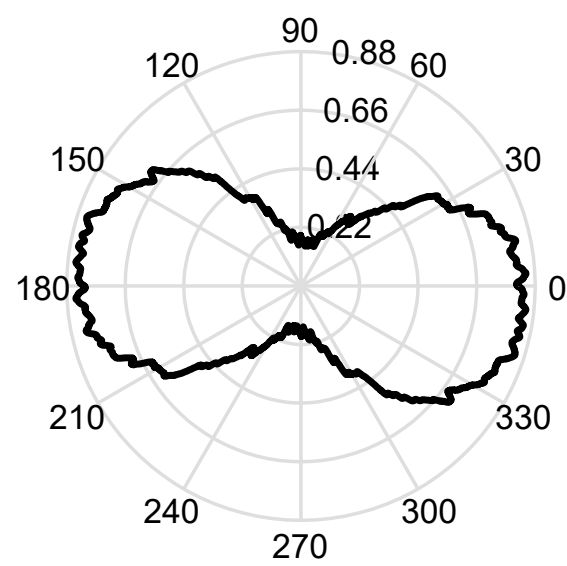

(c)

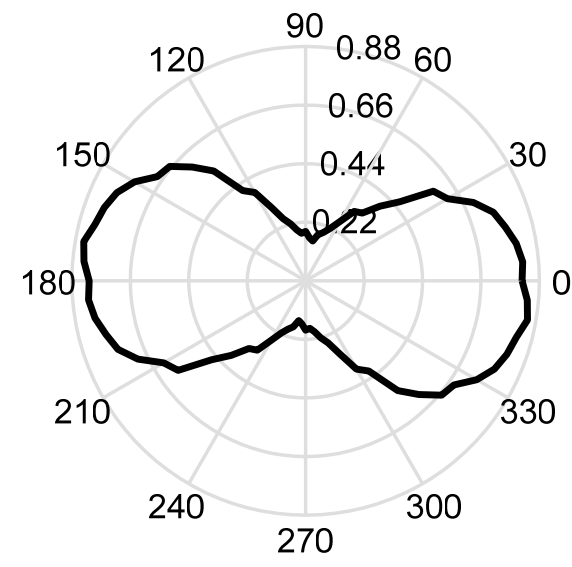

(e)

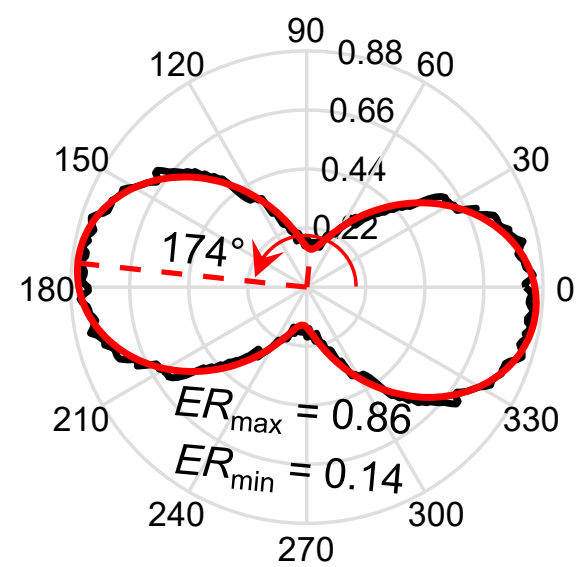

(b)

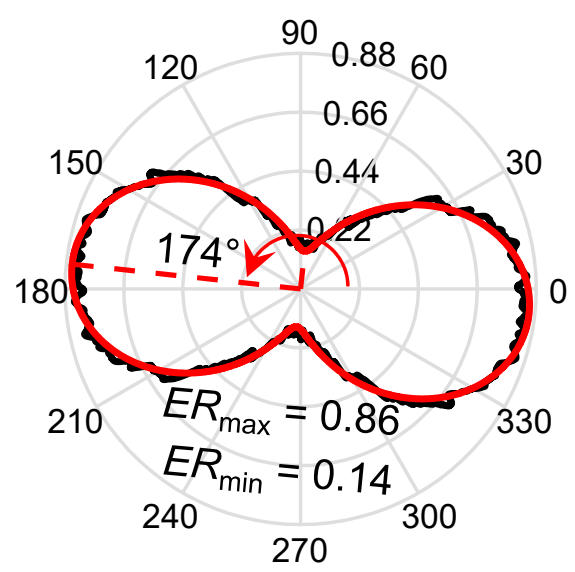

(d)

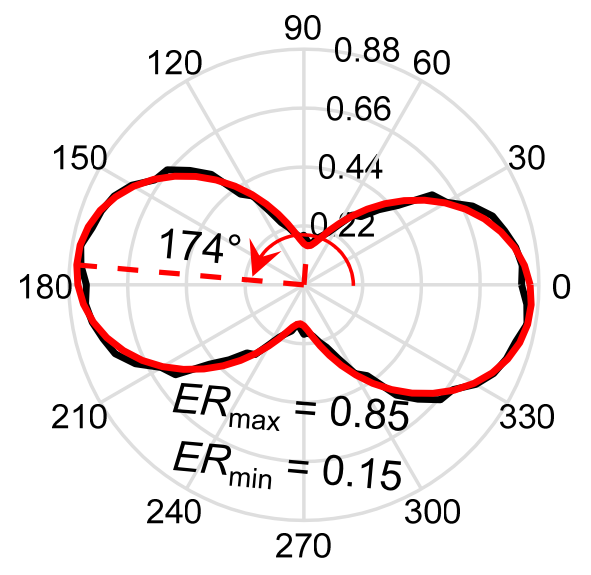

(f) 


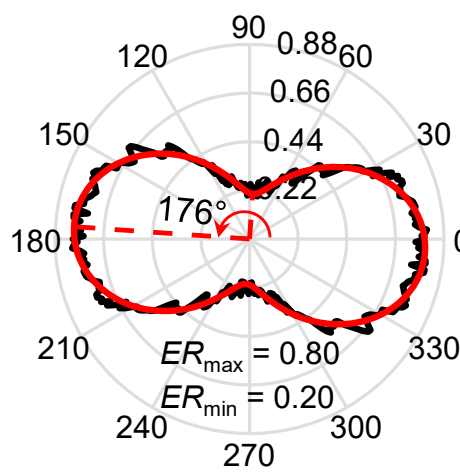

(a) $P P S=2$ pixels



(d) $P P S=14$ pixels

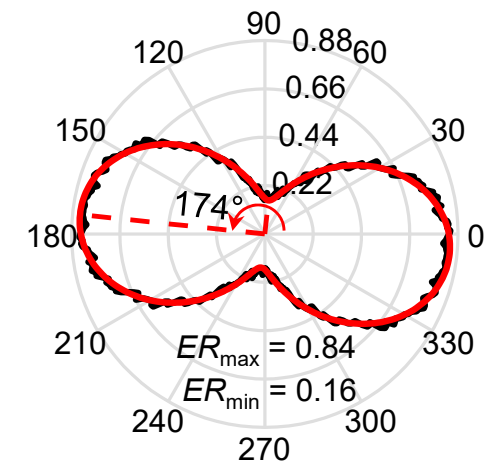

(b) $P P S=4$ pixels



(e) $P P S=28$ pixels

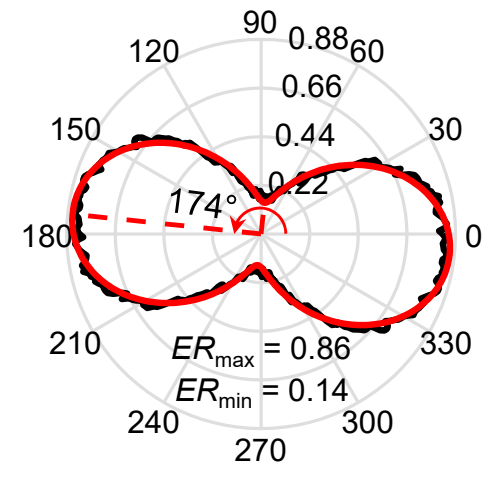

(c) $P P S=7$ pixels

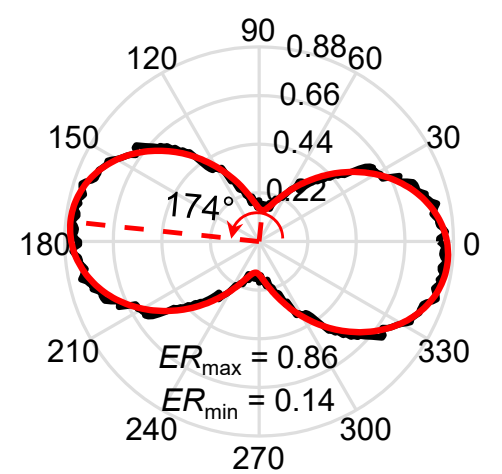

(f) $P P S=110$ pixels

Original ER plot Fourier ER plot 


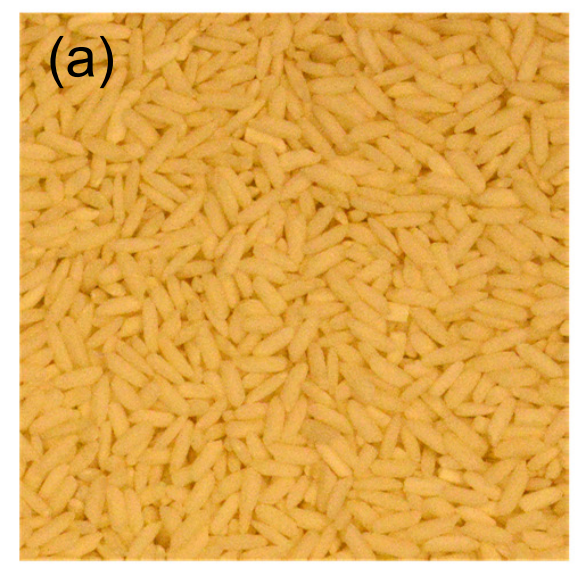

(b) $\begin{array}{cc}120 \quad & 90 \\ 0.88 & 0.66\end{array}$


(d) $120 \quad 900.8860$
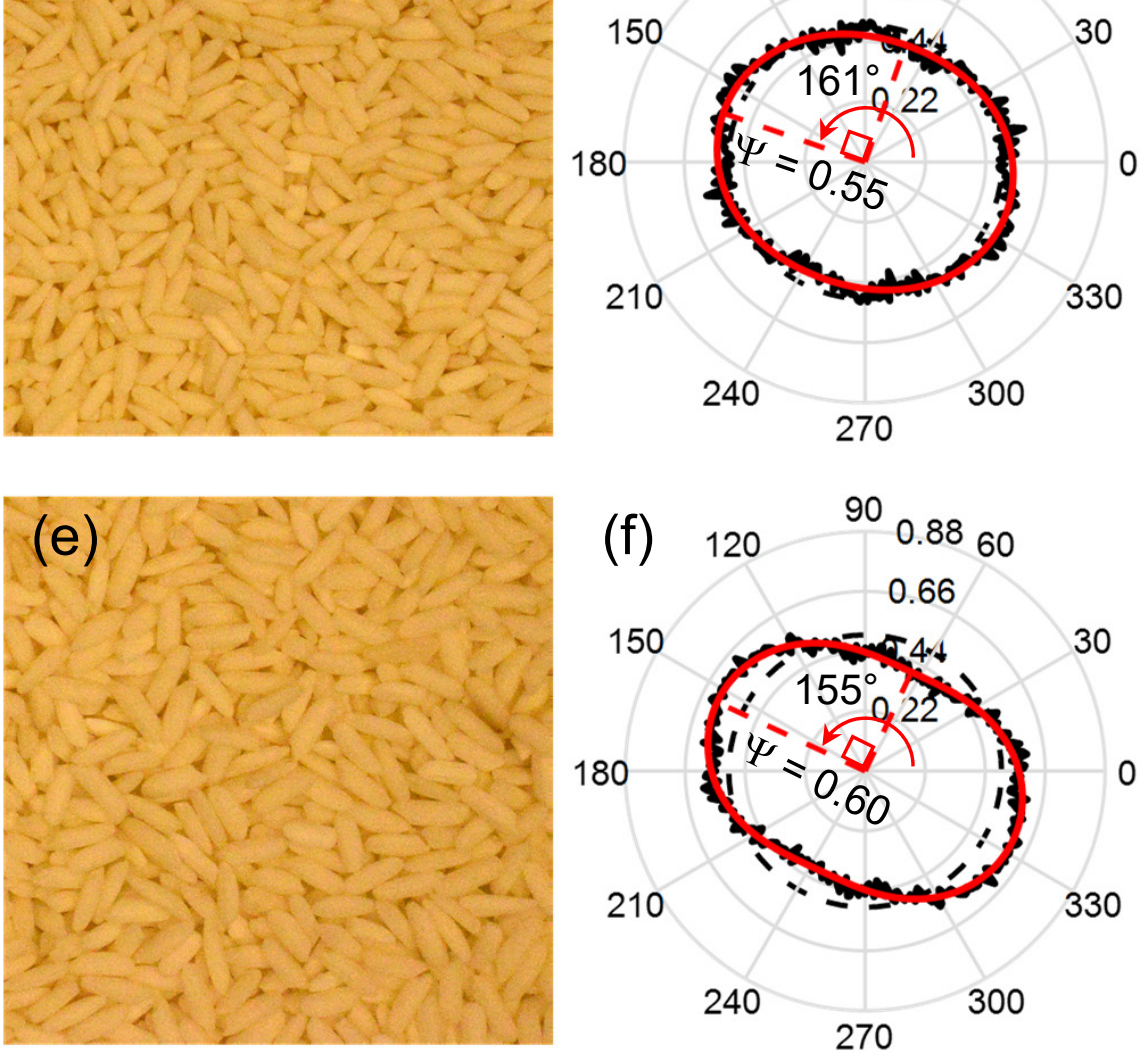

(f) $\begin{array}{cc}120 \quad & 0.88 \\ 0.66 & 60\end{array}$



- Fourier ER plot

Original ER plot

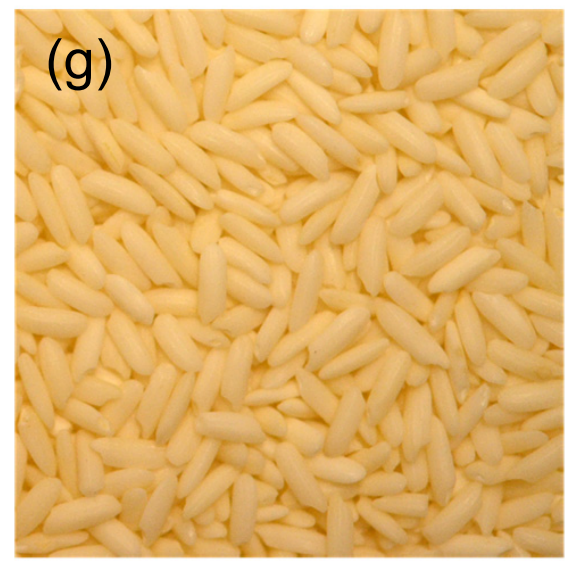

(h) $\begin{array}{cc}120 \quad & 0.88 \\ 0.66 & 60\end{array}$
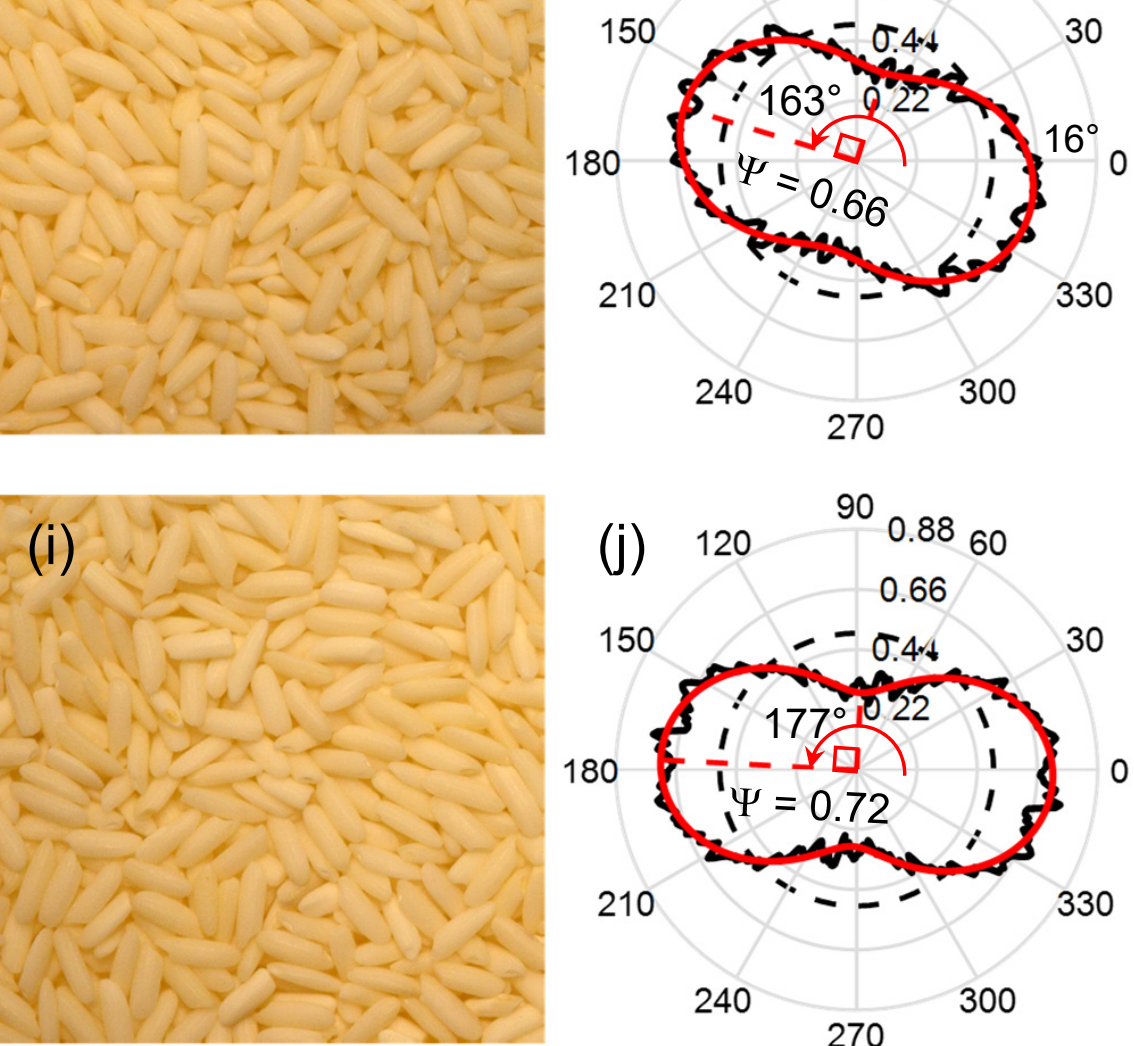

(j) $120 \quad{ }_{0.66}^{90} 0.88$

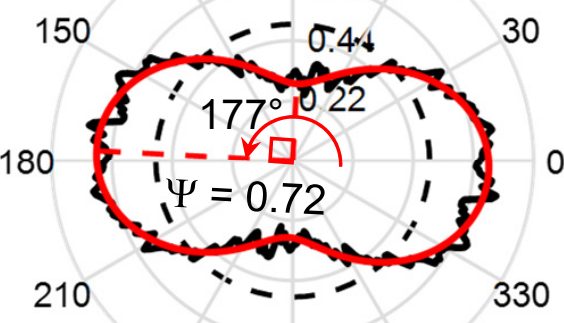

$240 \quad 270 \quad 300$



(I) $\begin{array}{cc}120 & 90.88 \\ 0.66\end{array}$

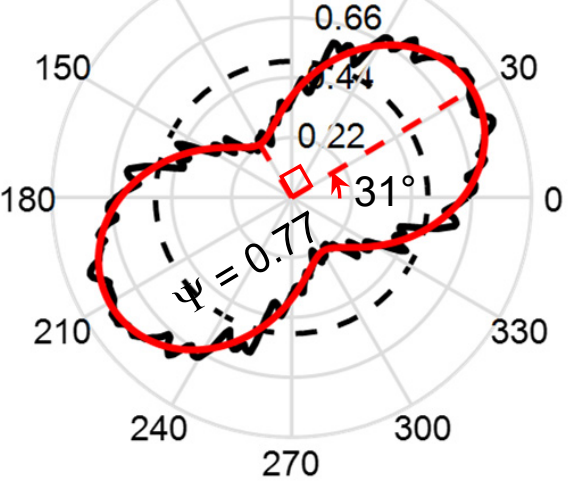




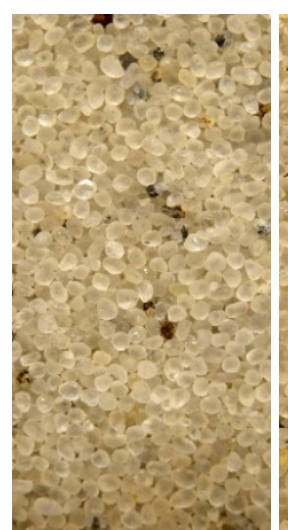

(a)

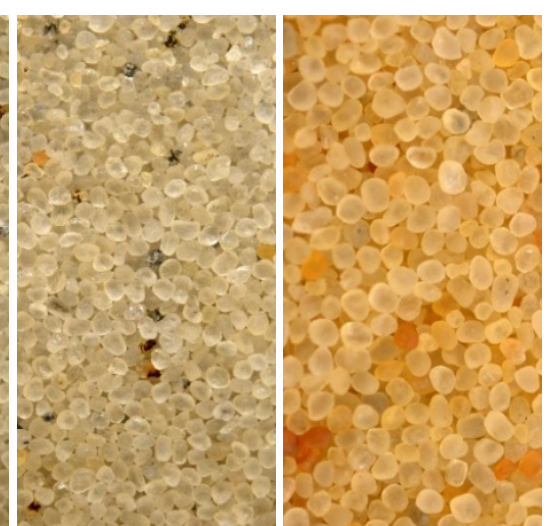

(b)

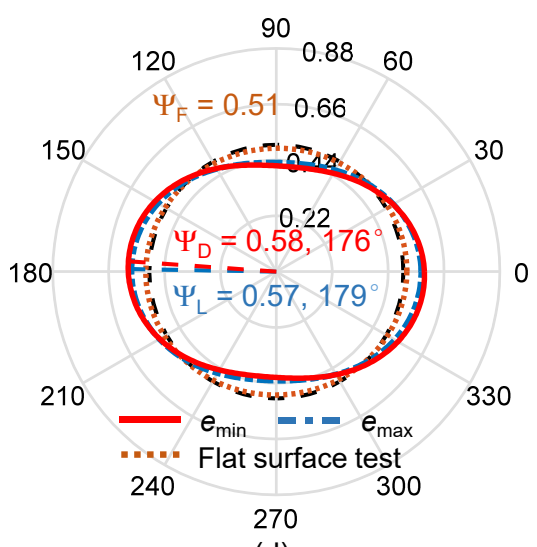

(d) 




(a) (b) (c)

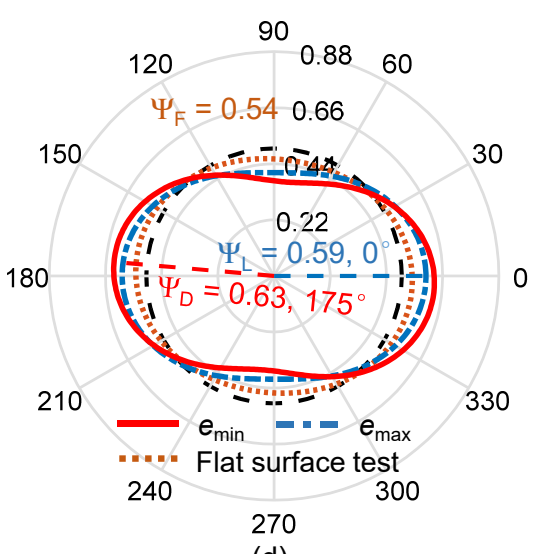

(d) 




(a) (b)

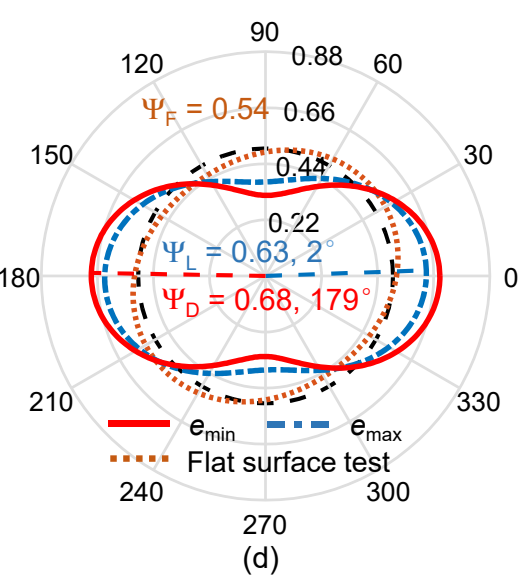

(d) 


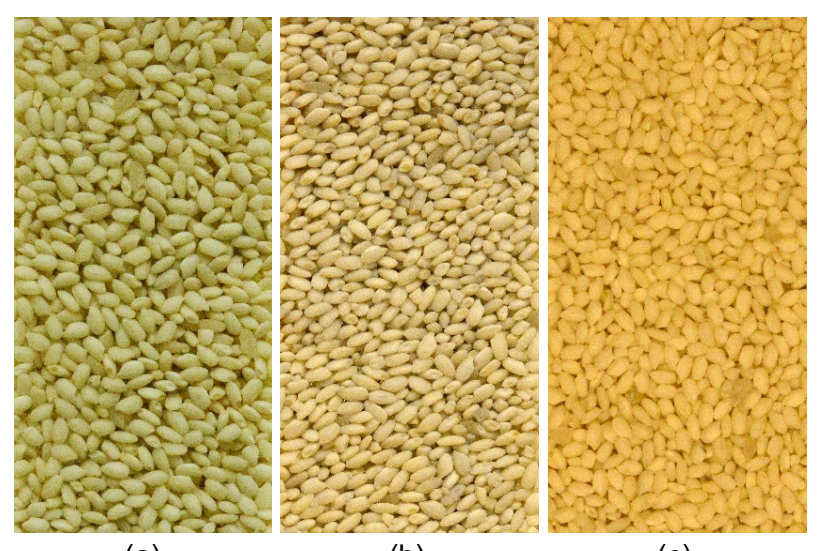

(a) (b)

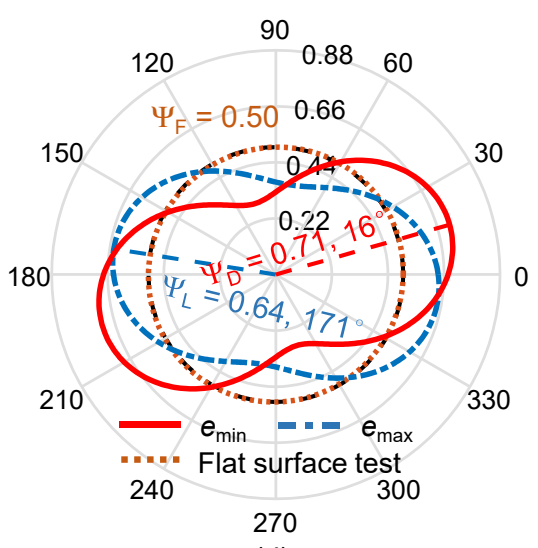

(d) 


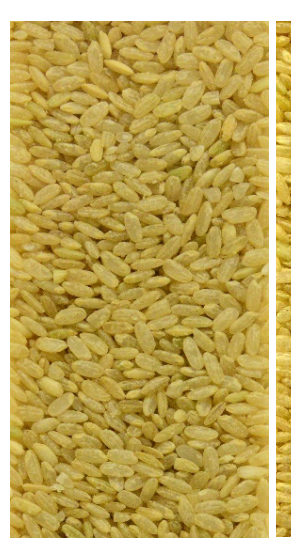

(a)

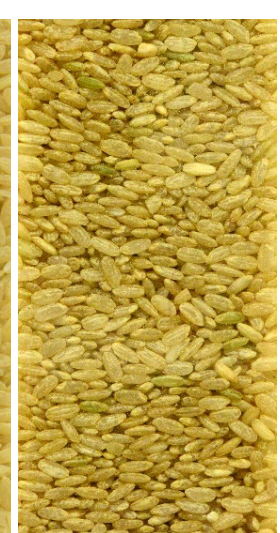

(b)

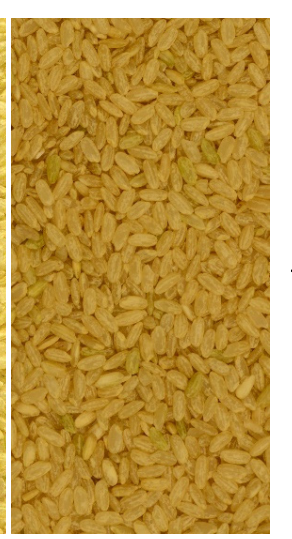

(c)



(d) 


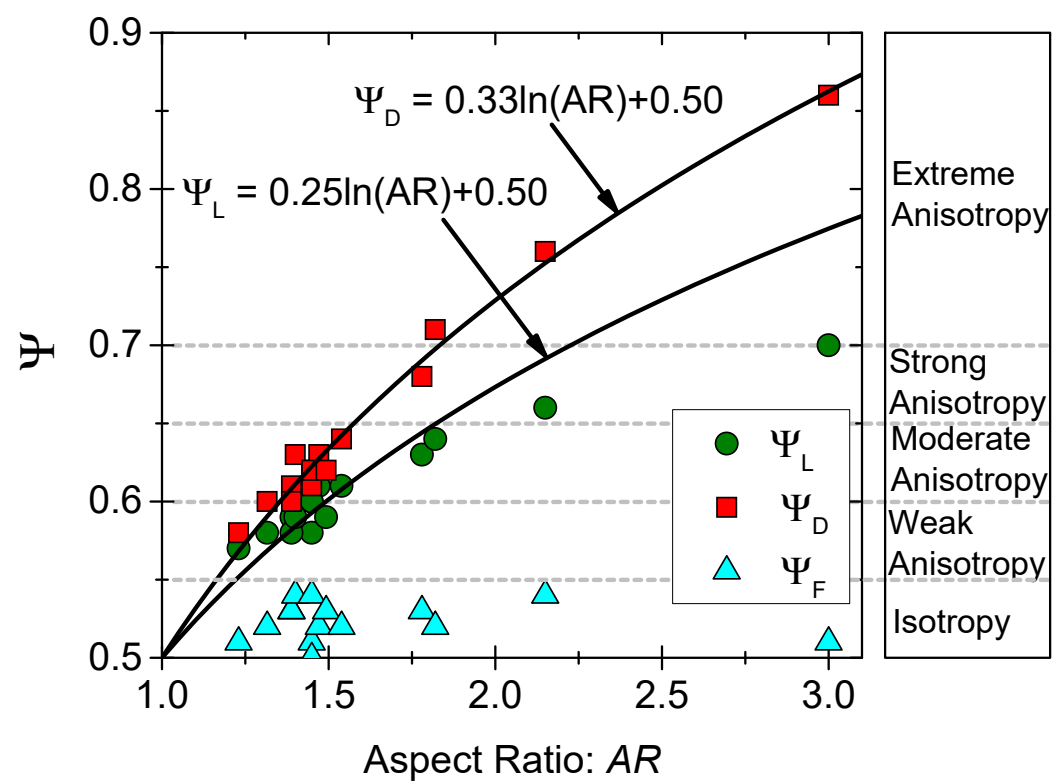



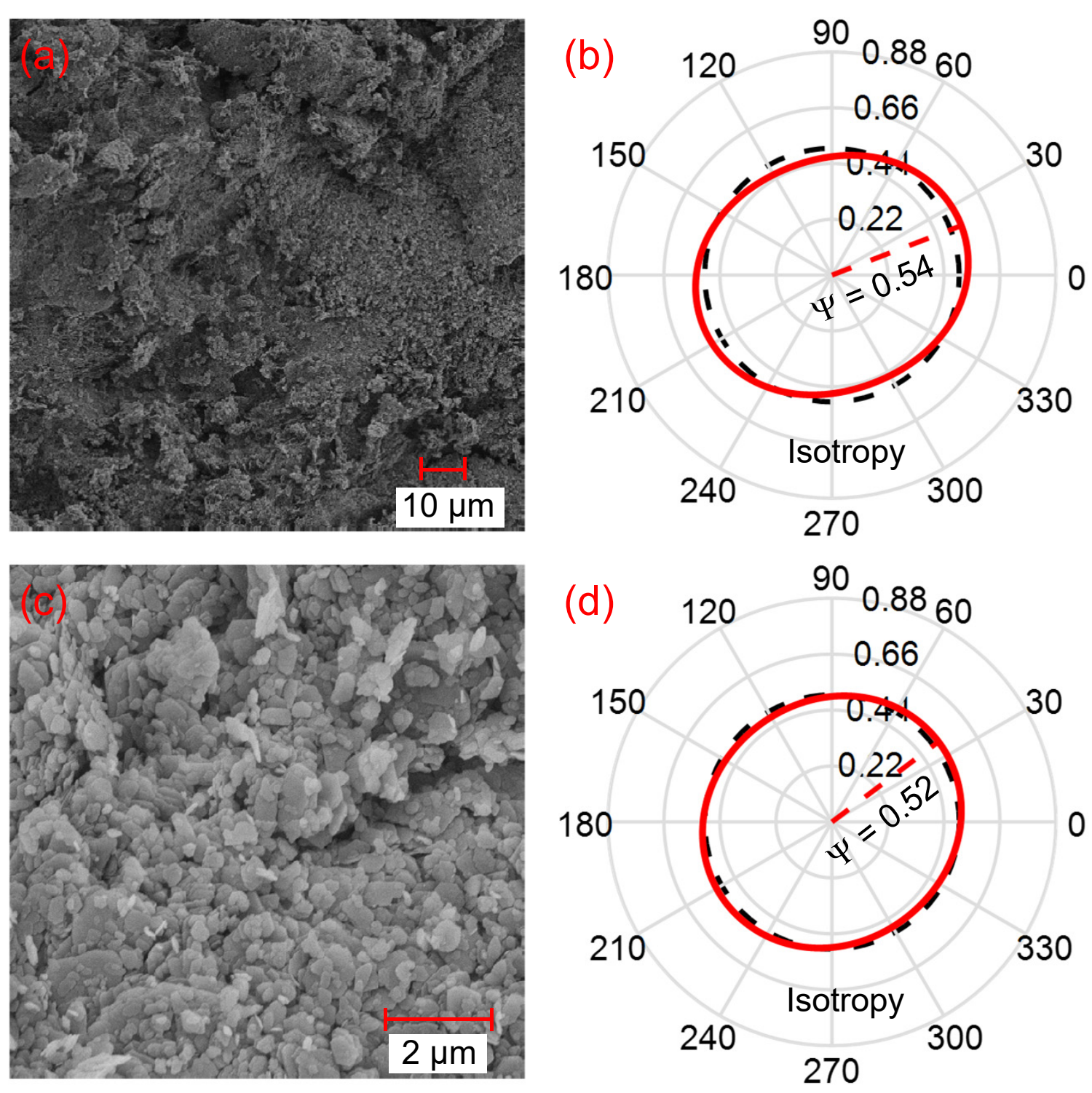

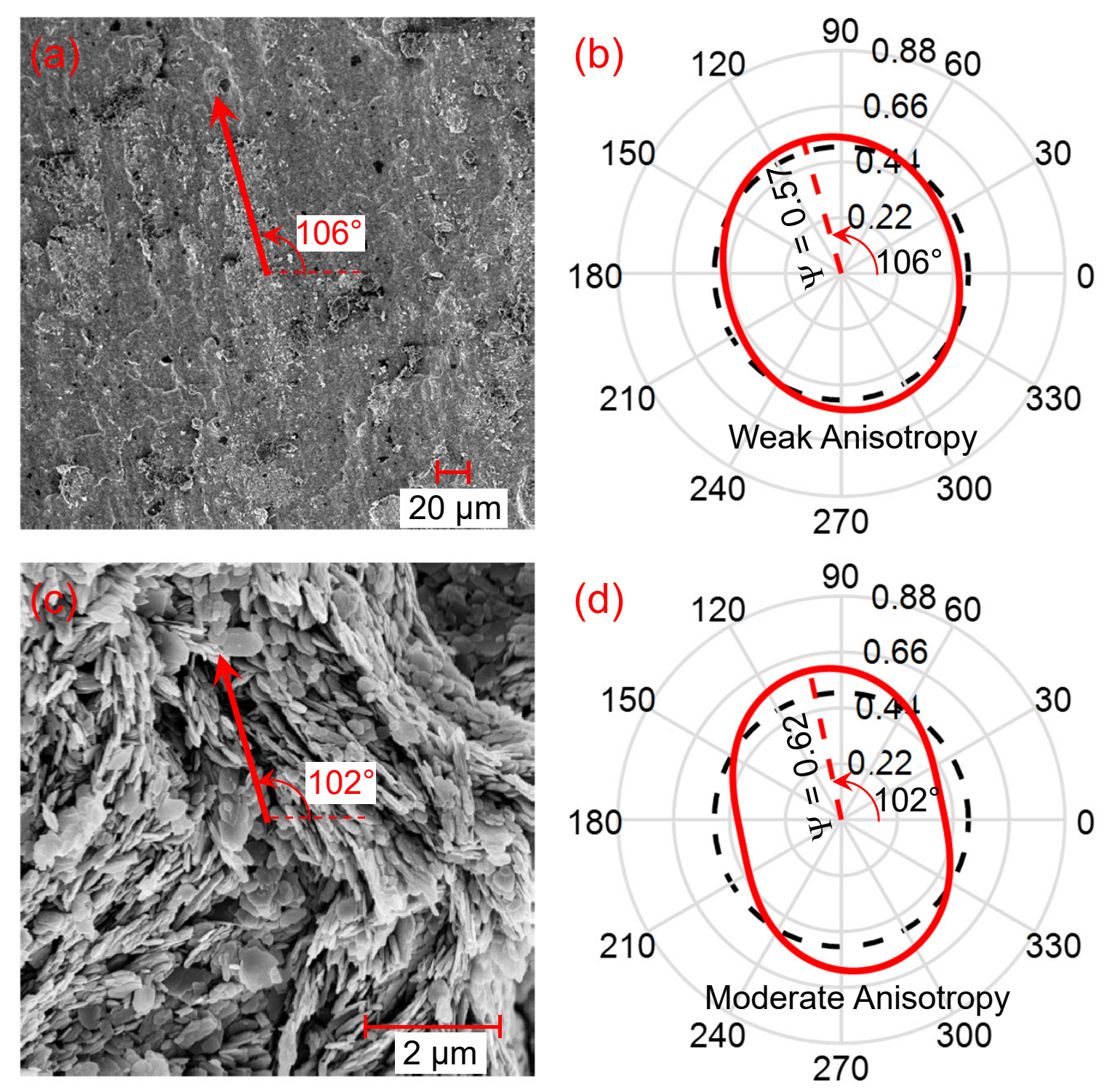

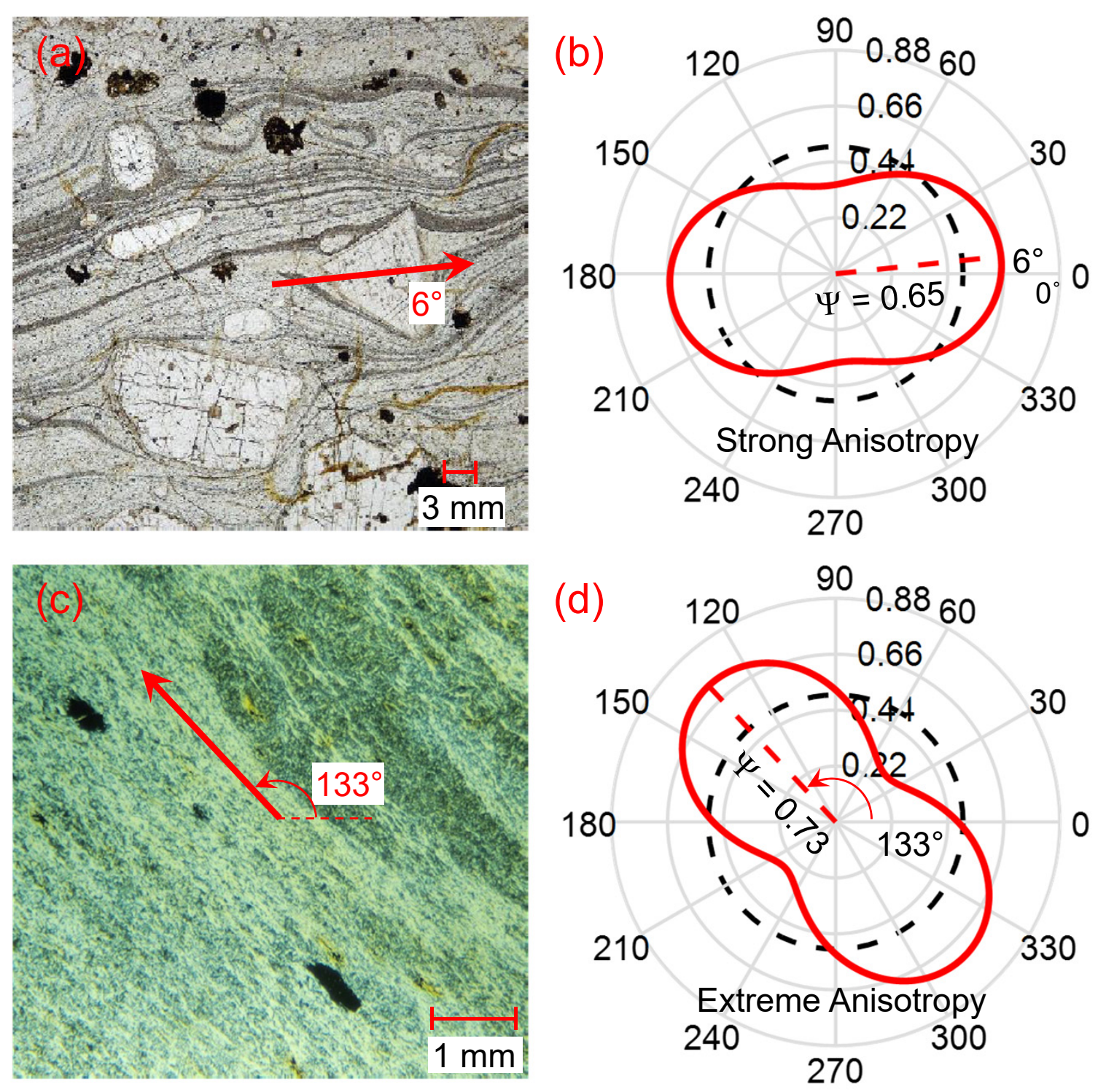


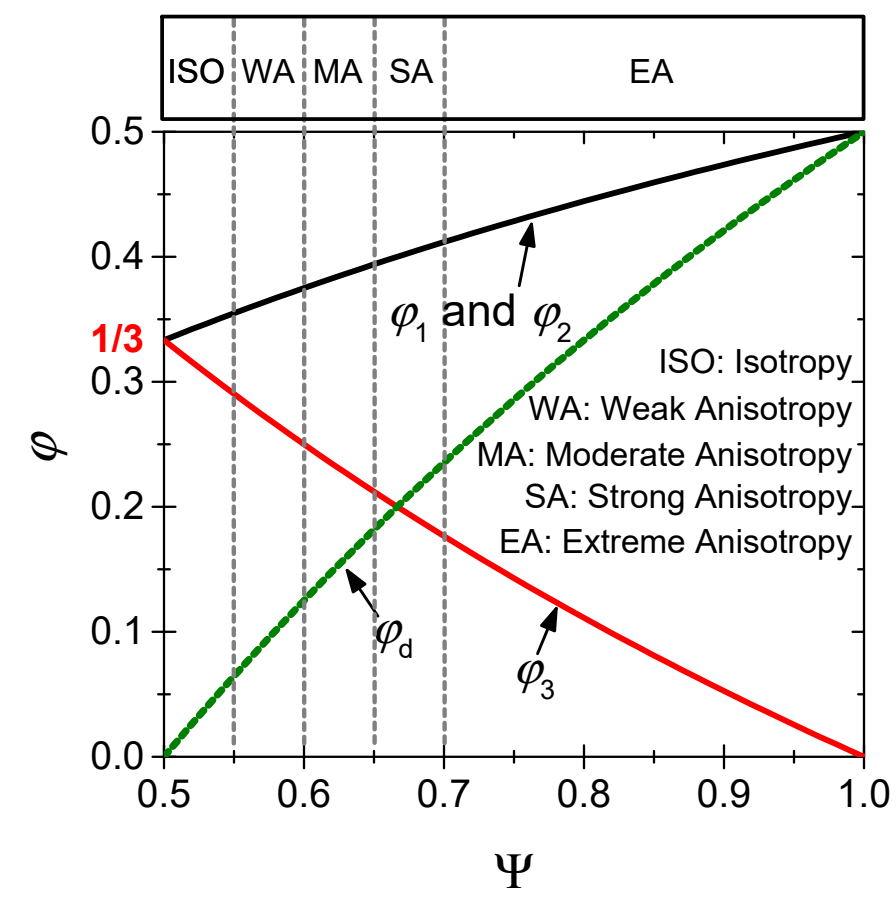

Figure 22 